\title{
Asymptotic Performance of Energy Detector in Fading and Diversity Reception
}

\author{
Vesh Raj Sharma Banjade, Student Member, IEEE, Chintha Tellambura, Fellow, IEEE, and \\ Hai Jiang, Senior Member, IEEE
}

\begin{abstract}
Missed-detection probability expressions for energy detectors often involve infinite series and do not provide quick insights into the effects of operating conditions. To overcome these limitations, we develop novel asymptotic analyses by proposing an approximate probability density function (PDF) of a random variable $\beta$, which, in general, can characterize fading channels in diverse operating conditions. The coefficients of the proposed approximate PDF of $\beta$ are obtained by matching the coefficients of the approximate PDF's series expansion (or coefficients of the approximate PDF's moment generating function (MGF)) with those of the exact PDF (or MGF) of $\beta$. By using the proposed approximation, a unified closed-form asymptotic missed-detection probability is derived. Its usefulness is then demonstrated for fading channels without and with antenna diversity, for cooperative detection, and in co-channel interference. For each case, the sensing gain, which reveals the effect of the operating conditions on the detection performance, is determined explicitly. Furthermore, the asymptotic complementary area under the receiver operating characteristic curve, an alternative performance metric, is derived, and found to reveal the sensing gain. Numerical results verify the accuracy of our derived asymptotic expressions over a wider signal-to-noise ratio (SNR) range compared to the existing asymptotic solution, which is accurate only for high SNRs.
\end{abstract}

Index Terms-Diversity combining, energy detector, fading, false alarm probability, missed-detection probability, moment generating function.

\section{INTRODUCTION}

$\mathbf{T}$ HE exponential growth of mobile data traffic is demanding more and more radio frequency (RF) spectrum, which although being scarce, is underutilized across space and time. Such situation necessitates the coexistence of multiple wireless networks. To support such coexistence, it is critical to determine whether or not users (for example, licensed users operating in the 54-806 MHz TV band) are operating in a given portion of the RF spectrum. For this purpose, the energy detector (ED) has attracted massive wireless research interest due to its simple structure and low hardware complexity [1]. The ED performance is fundamentally measured by the false alarm probability,

Manuscript received November 14, 2014; revised March 21, 2015; accepted April 30, 2015. Date of publication May 8, 2015; date of current version June 12, 2015. Part of this work was presented at the IEEE Global Communications Conference (GLOBECOM), Austin, TX, USA, December 2014. This work was supported by the Natural Sciences and Engineering Research Council (NSERC) of Canada. The associate editor coordinating the review of this paper and approving it for publication was J. Yuan.

The authors are with the Department of Electrical and Computer Engineering, University of Alberta, Edmonton, AB T6G 2V4, Canada (e-mail: sharmaba@ualberta.ca; chintha@ece.ualberta.ca; hai1@ualberta.ca).

Color versions of one or more of the figures in this paper are available online at http://ieeexplore.ieee.org.

Digital Object Identifier 10.1109/TCOMM.2015.2431259
$P_{f}$, and the detection probability, $P_{d}$, or, equivalently, the missed-detection probability $P_{m d}=1-P_{d}$. However, in fading channels, the average $P_{m d}\left(P_{d}\right)$ denoted by $\bar{P}_{m d}\left(\bar{P}_{d}\right)$ is the critical measure. The process of determining $\bar{P}_{m d}\left(\bar{P}_{d}\right)$ involves two steps. First, $P_{m d}(\gamma)\left(P_{d}(\gamma)\right)$ for a fixed channel realization is given in terms of the Marcum- $Q$ function (see (2) in Section II), which depends on the random (instantaneous) signal-to-noise ratio (SNR) $\gamma$. By definition, $\gamma$ can be expressed as $\gamma=\bar{\gamma} \beta$, where $\bar{\gamma}$ is the average SNR (referred to as "SNR" henceforth), ${ }^{1}$ and $\beta$ is a non-negative random variable, which in turn depends on the operating conditions (including the fading channel, antenna diversity, co-operative diversity, interference, and others). Thus, $\beta$ is characterized by a probability density function (PDF) $f(\beta)$. In the second step, $P_{m d}(\gamma)\left(P_{d}(\gamma)\right)$ is integrated over $f(\beta)$ to obtain the average detection performance (see details in Section II).

This process has been extensively used for accurately characterizing the ED performance in a wide variety of operating conditions [2]-[7]. Although these results are exact (without any approximations), they have some limitations. For example,

(i) The multiple-antenna based results in [2] and [3] are restricted to the Rayleigh fading model only, due mainly to the intractability of Marcum- $Q$ integrals, in general.

(ii) The results for Nakagami- $m$ and Rician fading derived by utilizing contour-integral representation of the Marcum- $Q$ function consist of complicated higherorder derivatives of composite functions [5].

(iii) The unified expressions presented in [6] and [7] involve infinite series, which require truncation (to finite terms). However, tight bounds on the truncation error are often analytically intractable, and hence, trial-and-error methods are needed to determine the truncation point for the desired level of precision.

(iv) None of the derived expressions provide quick insights into the impact of the operating conditions, but require numerical analysis to reveal such insights.

These limitations, however, can be mitigated by using asymptotic techniques. Before discussing their advantages, we will briefly elaborate on the meaning of the term "asymptotic." For example, $1 / x$ is an asymptotic of $1 /(x+1)$ because $\lim _{x \rightarrow \infty} \frac{x}{x+1}=1$. More specifically, if $x \rightarrow 0^{+}$( $x$ tends to 0 from above), then the notation $h(x)=g(x)+O\left(x^{N+1}\right)$, where $g(x)=\sum_{n=0}^{N} a_{n} x^{n}$, implies that the difference $|h(x)-g(x)|$

\footnotetext{
${ }^{1}$ For brevity, in the remainder of this paper, the term "SNR" without "instantaneous" means the "average SNR."
} 
is smaller than $C\left|x^{N+1}\right|$ for a constant $C$ as $x \rightarrow 0^{+}$. Then, $g(x)$ is called an asymptotic (approximation) of $h(x)$ with an error term $O\left(x^{N+1}\right)$ as $x \rightarrow 0^{+}$. Similarly, for $x \rightarrow \infty$, a series of $x^{-n}$ forms an asymptotic expansion. Thus, for an ED, if $\bar{P}_{m d}^{\text {exact }}$ is the exact missed-detection probability, and $\bar{P}_{m d}^{\text {asy }}$ is an approximation at high $\operatorname{SNR}(\bar{\gamma} \gg 1)$, assume

$$
\bar{P}_{m d}^{\text {exact }}=\bar{P}_{m d}^{\text {asy }}+O\left(\frac{1}{\bar{\gamma}^{M}}\right)
$$

where $M$ is a non-negative integer. Then, $\bar{P}_{m d}^{\text {asy }}$ is called an asymptotic (approximation) of $\bar{P}_{m d}^{\text {exact }}$ with error term $O\left(1 / \bar{\gamma}^{M}\right)$. Moreover, since the error term decays as $1 / \bar{\gamma}^{M}$, for sufficiently large $\bar{\gamma}$, the accuracy of $\bar{P}_{m d}^{\text {asy }}$ is good enough for all practical engineering applications. Hence, to derive such $\bar{P}_{m d}^{\text {asy }}$, we first note that as the SNR becomes large $(\bar{\gamma} \gg 1)$, detection errors occur only if the channel fades deeply. Since a deep fading is equivalent to $\beta \rightarrow 0^{+}$, a suitable polynomial representation of $f(\beta)$ near $\beta=0$ can be used to derive the average asymptotic missed-detection probability $\bar{P}_{m d}^{\text {asy }}$.

Despite having such advantages, the existing asymptotic analysis of ED is limited only to [8]. This work utilizes the simple, approximate analysis presented in the seminal work by Wang and Giannakis [9] to derive the asymptotic $\bar{P}_{m d}$, which is accurate for the high SNR regime $(\bar{\gamma} \gg 1)$. The results of [8] are important for determining the "sensing gain," which is the magnitude of the slope of the log-log plot of $\bar{P}_{m d}$ vs. $\bar{\gamma}$ at high SNR. More importantly, the sensing gain provides quick insights into how fading impacts the ED, and thus is a useful performance indicator.

However, the asymptotic analysis [8] is inherently limited to the high SNR regime (say, $\bar{\gamma} \geq 20 \mathrm{~dB}$ ). In practice, the operating SNR can be well below $20 \mathrm{~dB}$, in, for example, the IEEE 802.16 local and metropolitan area networks [10]. Thus, the analysis in [8] is not sufficient for characterizing the ED performance over a wider SNR range (optimistically, $0 \leq \bar{\gamma} \mathrm{dB}<\infty$ ). Moreover, a unified expression for $\bar{P}_{m d}$, which would be applicable to a multitude of wireless communication scenarios thus providing an effective platform for designing practical detection systems, is needed. However, such a platform is lacking. To address these requirements, we propose a new simple approximation for the PDF of $\beta$ as $\beta \rightarrow 0^{+}$and derive the corresponding asymptotic $\bar{P}_{m d}$. The attractive features of the results derived by using our proposed analysis are the following:

1) Unified expressions for multipath fading channels without and with antenna diversity, with multiple cooperative relays, with interferers, and in other operating conditions;

2) Explicit sensing gain expressions;

3) Closed-form expressions without infinite-series sums or higher order derivatives; and

4) High accuracy over wider SNR ranges.

These features are achieved by our proposed approximation $f^{\mathrm{app}}(\beta)(6)$, whose parameters can be obtained from the operating conditions by matching $f^{\text {app }}(\beta)$ to the exact $f(\beta)$ or by matching the moment generating function (MGF) of $\beta$ obtained by using $f^{\text {app }}(\beta)$ to that obtained by using the exact $f(\beta)$ (see details in Section III). Besides these benefits, performance metrics other than $\bar{P}_{m d}$ can also be analyzed. For example, we derive a unified asymptotic expression for the average complementary area under the receiver operating characteristic (ROC) curve (CAUC), which serves as a single figure of merit for characterizing the ED performance [11].

The rest of this paper is organized as follows. The background of energy detection is briefly discussed in Section II. The new approximation for the exact PDF of $\beta$ is proposed in Section III. The unified asymptotic $\bar{P}_{m d}$ is derived in Section IV. Analyses for fading channels without diversity, with antenna diversity, with cooperative diversity, and in interference are presented in Section V, Section VI, Section VII, and Section VIII, respectively. The asymptotic expression for the average CAUC is derived in Section IX. Concluding remarks are made in Section X.

Notations: $\mathbb{P}(\cdot), \mathbb{E}[\cdot], \mathcal{M}_{\beta}(s)=\mathbb{E}\left[e^{-s \beta}\right]$, and $J$ denote the probability of an event, mathematical expectation, moment generating function of $\beta$, and imaginary unit $(\sqrt{-1})$, respectively.

\section{Problem Statement}

The ED filters the observed signal over a bandwidth $W$, prefiltering the noise. This process is followed by squaring and integration over a period $T$ to yield a measure of its energy $Y$, which is then compared against a detection threshold $\lambda$ to determine the presence or absence of the transmit signal under test (SUT). We denote the presence and absence of the SUT by using hypotheses $H_{1}$ and $H_{0}$, respectively. Based on the sampling theorem approximation, given $2 u$ samples $(u=T W$ is called the time-bandwidth product [2]), the false alarm probability $P_{f}=\mathbb{P}\left(Y>\lambda \mid H_{0}\right)$ and the missed-detection probability $P_{m d}=1-\mathbb{P}\left(Y>\lambda \mid H_{1}\right)$ have well-known forms as [2]

$$
\begin{aligned}
P_{f} & =\frac{\Gamma(u, \lambda / 2)}{\Gamma(u)}, \\
P_{m d}(\gamma) & =1-Q_{u}(\sqrt{2 \gamma}, \sqrt{\lambda}),
\end{aligned}
$$

where, recalling that the instantaneous SNR is expressed as $\gamma=\bar{\gamma} \beta$, with $\beta$ being the random variable depending upon the operating conditions ( $\bar{\gamma}$ being the average $\mathrm{SNR}), Q_{u}(\cdot, \cdot)$ is the $u$-th order generalized Marcum- $Q$ function [12], $\Gamma(c, v)=$ $\int_{v}^{\infty} x^{c-1} e^{-x} d x$ is the upper-incomplete Gamma function, and $\Gamma(c)=\Gamma(c, 0)$ is the Gamma function [13]. Clearly, the false alarm probability (1) is independent of $\beta$ while the misseddetection probability (2) depends upon $\beta$ (as $\gamma=\bar{\gamma} \beta$ ) and needs to be averaged over $f(\beta)$ for evaluating the overall (average) performance, as shown next.

\section{A. Performance of Energy Detector Over Fading Channels}

The average probability of missed-detection, denoted as $\bar{P}_{m d}$, is the expectation of (2) over $f(\beta)$ which can be expressed as

$$
\bar{P}_{m d}=\mathbb{E}_{\beta}\left[P_{m d}(\gamma)\right]=1-\int_{0}^{\infty} Q_{u}(\sqrt{2 \bar{\gamma}} \beta, \sqrt{\lambda}) f(\beta) d \beta,
$$

where $\mathbb{E}_{\beta}[\cdot]$ denotes the expectation with respect to $\beta$. Thus, the main challenge in (3) is averaging the Marcum- $Q$ function over $f(\beta)$. Since closed-form solutions for integrals involving the Marcum- $Q$ function are very limited [12], it is important to find 
an approximate form of $f(\beta)$ that facilitates the evaluation of (3) without leading to tedious analytical expressions involving complicated special functions and/or infinite series.

\section{B. Existing Approximation for $f(\beta)$}

To this end, the work [8] utilizes the approximate $f(\beta)$ proposed by Wang and Giannakis [9], to derive the asymptotic $\bar{P}_{m d}$. The main idea of [9] is that if the exact $\operatorname{PDF} f(\beta)$ can be expanded as a Taylor's series as $\beta \rightarrow 0^{+}$(Maclaurin's series) in the form

$$
f(\beta)=\sum_{i=0}^{\infty} a_{i} \beta^{t+i},
$$

where $a_{i}$ for $i=0,1,2, \ldots$ are coefficients in the expansion, and $t \geq 0$ represents the order of smoothness of $f(\beta)$ at $\beta=0$, then $f(\beta)$ can be approximated by a monomial [9] as

$$
f^{\mathrm{wg}}(\beta)=a \beta^{t}+O\left(\beta^{t+1}\right),
$$

where the parameters $a$ and $t$ in (5), which depend on the operating conditions, are obtained by matching the first nonzero term in (4) with (5). Here, we use the superscript "wg" to indicate "Wang and Giannakis," the authors of [9]. Note that the expansion (4) holds for many practical fading models like Rayleigh, Nakagami- $q$, Nakagami- $n$ and Nakagami- $m$ [9].

By utilizing $f^{\mathrm{wg}}(\beta)$, the authors in [8] derive an asymptotic $\bar{P}_{m d}$ and show that for large SNRs $(\bar{\gamma} \gg 1)$, the sensing gain is equal to $t+1$. However, their $\bar{P}_{m d}$ is accurate only in the high SNR regime (say, $\bar{\gamma} \geq 20 \mathrm{~dB}$ ). Intuitively, this result suggests that an approximation for $f(\beta)$ other than $f^{\mathrm{wg}}(\beta)$ is needed that can yield a closed-form $\bar{P}_{m d}$ which is accurate over a wider range of SNRs (say, $0 \leq \bar{\gamma} \mathrm{dB}<\infty$ ). Furthermore, the derived $\bar{P}_{m d}$ at high SNR should also reveal the sensing gain. With these goals in mind, we propose a new approximation for the exact $f(\beta)$ in the following section.

\section{NeW ApProXimation fOR $f(\beta)$}

The existing approximation (5) utilizes a single term in (4) and is accurate only for high SNRs. One way to achieve a better accuracy over wider SNR ranges may be to utilize more number of terms in (4). However, simply considering more number of terms in (4) may still lead to a diverging behavior of the approximate $f(\beta)$ at $\beta \rightarrow \infty$ (similar to $f^{\mathrm{wg}}(\beta)$ curve shown in Fig. 1) which may further lead to the average (over $0 \leq \beta<\infty) P_{m d}$ to exceed 1 for small SNRs (as explained in Section III-B). Thus, in addition to improving the accuracy, it is also necessary to ensure that the approximate $f(\beta)$ does not diverge as $\beta \rightarrow \infty$. Additionally, the approximate $f(\beta)$ should facilitate a closed-form evaluation of (3).

\section{A. Proposed Approximation}

To this end, since an exponential function of the form $e^{-\beta}$ would converge as $\beta \rightarrow \infty$, we consider a new representation of $f(\beta)$ by combining (5) with a dual exponential sum to propose an approximation $f^{\text {app }}(\beta)$ of the form

$$
f^{\text {app }}(\beta)=a \beta^{t}\left(e^{-\theta_{1} \beta}+e^{-\theta_{2} \beta}\right), \text { as } \beta \rightarrow 0^{+},
$$

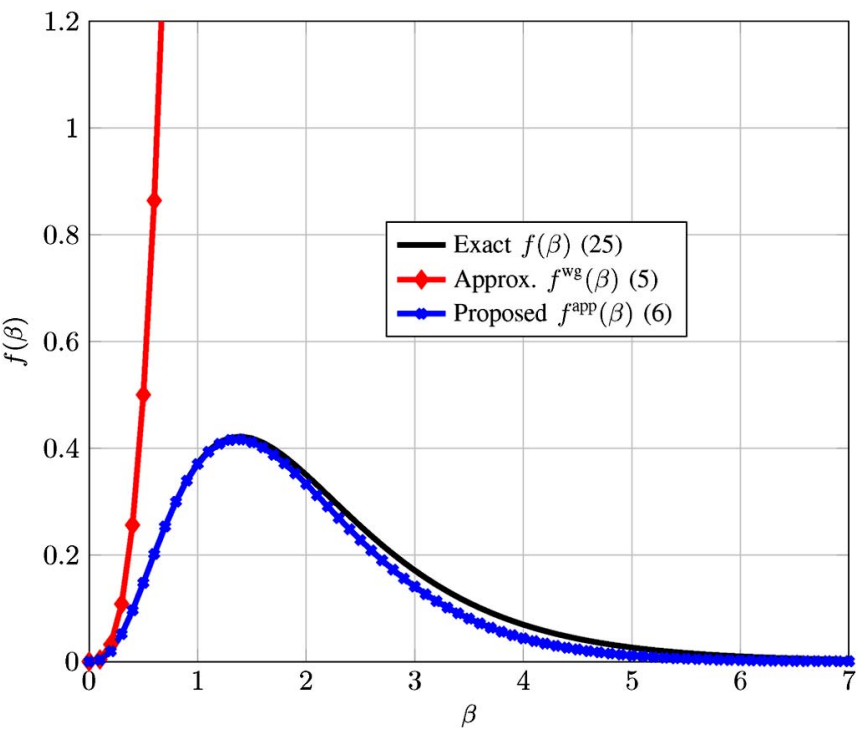

Fig. 1. $f^{\text {app }}(\beta), f^{\mathrm{wg}}(\beta)$, and exact $f(\beta)$ for 4-branch SC in i.i.d. Rayleigh fading.

where $a>0, t$ is a non-negative integer, $\theta_{1} \geq 0$, and $\theta_{2} \geq 0$ are the parameters that can be chosen to match the operating conditions (fading, antenna diversity, cooperative diversity, etc.). Interestingly, the Maclaurin's series expansion of (6) satisfies (4) (details in Section III-B). Note that $f^{\mathrm{wg}}(\beta)$ is a special case of our $f^{\text {app }}(\beta)$ when $\theta_{1}=\theta_{2}=0$. Also, $f^{\text {app }}(\beta)$ is equivalent to [14, eq. (3)] for the case $\theta_{1}=\theta_{2}=\theta$. Thus, $f^{\text {app }}(\beta)$ is more general than either $f^{\mathrm{wg}}(\beta)$ or [14, eq. (3)]. Moreover, $f^{\mathrm{app}}(\beta)$, just like $f^{\mathrm{wg}}(\beta)$, is not a proper PDF; that is, the area under $f^{\text {app }}(\beta)$ is not necessarily 1 . Next, the parameters of $f^{\text {app }}(\beta)$ are derived from two methods based on the availability of the exact PDF or MGF of $\beta$.

\section{B. Parameter Determination Using Exact PDF of $\beta$}

In some cases of interest, the exact $f(\beta)$ is known analytically. Then, if its Maclaurin's series expansion follows (4), the parameters of $f^{\text {app }}(\beta)$ can be readily obtained as stated in Proposition 1.

Proposition 1: Given $a_{0}, a_{1}$, and $a_{2}$ from (4), the parameters of $f^{\text {app }}(\beta)$ can be expressed as

$$
\begin{aligned}
a & =\frac{a_{0}}{2} \\
\left(\theta_{1}, \theta_{2}\right) & =\left(\frac{b_{1}+\sqrt{2 b_{2}-b_{1}^{2}}}{2}, \frac{b_{1}-\sqrt{2 b_{2}-b_{1}^{2}}}{2}\right),
\end{aligned}
$$

with $b_{1}=-2 a_{1} / a_{0}$ and $b_{2}=4 a_{2} / a_{0}$.

Proof: By Maclaurin's series expanding (6) and grouping the resulting terms in ascending powers of $\beta$, we get

$$
f^{\text {app }}(\beta)=2 a \beta^{t}-\left(\theta_{1}+\theta_{2}\right) a \beta^{t+1}+\frac{\left(\theta_{1}^{2}+\theta_{2}^{2}\right)}{2} a \beta^{t+2}+O\left(\beta^{t+3}\right) .
$$

Then, by matching the coefficients of the first three terms in (4) with the corresponding coefficients in (9), the parameter $a$ in 
(6) is immediately given by (7) while the other two parameters, $\theta_{1}$ and $\theta_{2}$, satisfy the simultaneous equations

$$
\theta_{1}+\theta_{2}=-\frac{2 a_{1}}{a_{0}} \triangleq b_{1} \text { and } \theta_{1}^{2}+\theta_{2}^{2}=\frac{4 a_{2}}{a_{0}} \triangleq b_{2},
$$

which can be easily solved to yield (8).

To quickly gain insights into the benefits of $f^{\text {app }}(\beta)$, we compare it against $f^{\mathrm{wg}}(\beta)$ for selection combining (SC) in independent and identically distributed (i.i.d.) Rayleigh fading with $L=4$ antennas (Fig. 1). The exact $f(\beta)$ is given by (25), and the parameters of $f^{\text {app }}(\beta)$ are derived in Section VI-B. Fig. 1 reveals that $f^{\mathrm{wg}}(\beta)$ widely diverges from the exact $f(\beta)$ as $\beta$ gets large. Thus, $f^{\mathrm{wg}}(\beta)$ works only for $\beta \rightarrow 0^{+}$, which implies the deep fading condition. Thus $f^{\mathrm{wg}}(\beta)$ requires the operating SNR to be large $(\bar{\gamma} \gg 1)$ in order to yield low detection error probabilities. Furthermore, this result inherently suggests that when $f^{\mathrm{wg}}(\beta)$ is used in (3) for averaging the missed-detection probability, the resulting probability can even exceed 1 for small SNRs $\left(\bar{\gamma} \rightarrow 0^{+}\right)$. This finding indicates the breakdown of the asymptotic approach of [8] (demonstrated later in Sections IV-IX). In contrast, Fig. 1 shows that our proposed $f^{\text {app }}(\beta)$ follows the exact $f(\beta)$ more closely (without diverging) over $0 \leq \beta<\infty$. Thus, we can expect the resulting $\bar{P}_{m d}^{\text {asy }}$ expression to not break down even as $\bar{\gamma} \rightarrow 0^{+}$. These advantages of $f^{\text {app }}(\beta)$ have two causes:

(i) $f^{\text {app }}(\beta)$ matches the first three terms of the series expanded exact $f(\beta)$ (4) while $f^{\mathrm{wg}}(\beta)$ utilizes only the first term of (4). Thus, $f^{\text {app }}(\beta)$ uses more information from the exact $f(\beta)$, and this feature leads to highly accurate asymptotic results (see Section IV-A).

(ii) $f^{\text {app }}(\beta)$ does not diverge as $\beta \rightarrow \infty$ because of the factor $\left(e^{-\theta_{1} \beta}+e^{-\theta_{2} \beta}\right)$, a sum of two exponentials. This characteristic extends the valid SNR range of the resulting $\bar{P}_{m d}^{\text {asy }}$, thus maintaining reasonable accuracy even as $\bar{\gamma} \rightarrow 0^{+}$.

These advantages are numerically verified with some practical examples presented in Sections V-IX. Next, an alternative method to obtain the parameters of $f^{\text {app }}(\beta)$ based on the MGF of $\beta$ is presented.

\section{Parameter Determination Using the Exact MGF of $\beta$}

Proposition 1 is applicable only when the exact analytical $f(\beta)$ is known. However, $f(\beta)$ may be unknown or may not always be expressible as (4). On the other hand, if the exact MGF of $\beta$ is readily obtainable (for example, when $\beta$ is given by a sum of independent random variables, its MGF can readily be expressed as the product of MGFs of those random variables, and thus, the MGF may be easier to obtain than the corresponding $\mathrm{PDF})$, then the parameters of $f^{\mathrm{app}}(\beta)$ can be derived as follows.

Proposition 2: If the exact MGF of $\beta, \mathcal{M}_{\beta}(s), s>0$ is expandable in the series-form

$$
\mathcal{M}_{\beta}(s)=\frac{X_{0}}{s^{\alpha}}+\frac{X_{1}}{s^{\alpha+1}}+\frac{X_{2}}{s^{\alpha+2}}+O\left(\frac{1}{s^{\alpha+3}}\right),
$$

then as $s \rightarrow \infty$, the parameters $t$ and $a$ of $f^{a p p}(\beta)$ are given by

$$
t=\alpha-1, a=\frac{X_{0}}{2 \Gamma(\alpha)},
$$

with the parameters $\theta_{1}$ and $\theta_{2}$ satisfying

$$
\begin{aligned}
& \theta_{1}+\theta_{2}=-\frac{2 X_{1}}{X_{0} \alpha} \triangleq b_{1}, \\
& \theta_{1}^{2}+\theta_{2}^{2}=\frac{4 X_{2}}{X_{0} \alpha(\alpha+1)} \triangleq b_{2} .
\end{aligned}
$$

Then, substitution of $b_{1}$ and $b_{2}$ from (13) into (8) immediately yields $\theta_{1}$ and $\theta_{2}$.

Proof: From the initial value theorem for (one-sided) Laplace transforms, the behavior of $f(\beta)$ at $\beta \rightarrow 0^{+}$depends on its MGF at $s \rightarrow \infty$ [9]. Thus, the idea is to match the exact MGF of $\beta$ with the MGF obtained by using $f^{\text {app }}(\beta)$ as $s \rightarrow \infty$, provided the MGFs exist. The MGF corresponding to $f^{\operatorname{app}}(\beta)$, denoted by $\mathcal{M}_{\beta}^{\mathrm{app}}(s)$, can be obtained as

$$
\begin{aligned}
\mathcal{M}_{\beta}^{\mathrm{app}}(s) & =a \int_{0}^{\infty} e^{-s \beta} \beta^{t}\left(e^{-\theta_{1} \beta}+e^{-\theta_{2} \beta}\right) d \beta \\
& =a \Gamma(t+1)\left[\frac{1}{\left(s+\theta_{1}\right)^{t+1}}+\frac{1}{\left(s+\theta_{2}\right)^{t+1}}\right]
\end{aligned}
$$

where the definition of Gamma function $\Gamma(\cdot)$ is used. Expanding (14) in a series-form as $s \rightarrow \infty$, and re-arranging the terms, we get

$$
\begin{gathered}
\mathcal{M}_{\beta}^{\mathrm{app}}(s)=\frac{2 a \Gamma(t+1)}{s^{t+1}}-\frac{a(t+1) \Gamma(t+1)\left(\theta_{1}+\theta_{2}\right)}{s^{t+2}} \\
+\frac{a(t+1)(t+2) \Gamma(t+1)\left(\theta_{1}^{2}+\theta_{2}^{2}\right)}{2 s^{t+3}}+O\left(\frac{1}{s^{t+4}}\right) .
\end{gathered}
$$

Thus, matching (11) and (15) yields the parameters (12) and the two simultaneous equations in (13), which have the same form as (10) with the solution given by (8).

Having shown how the parameters of $f^{\text {app }}(\beta)$ are retrieved, we next derive the corresponding asymptotic missed-detection probability, $\bar{P}_{m d}^{\text {asy }}$.

\section{Average Missed-Detection Probability}

To derive $\bar{P}_{m d}^{\text {asy }}$, we need to substitute $f^{\text {app }}(\beta)$ into (3) and evaluate the resulting integral. However, direct evaluation of this integral would yield an infinite series expression [5, eq. (5)]. To eliminate this problem, we use the MGF $\mathcal{M}_{\beta}^{\text {app }}(s)(14)$ along with the contour integral representation of the Marcum- $Q$ function [15] to derive $\bar{P}_{m d}^{\text {asy }}$. Although such an approach has been utilized by [5], the results are given in terms of higher-order derivatives. In contrast, we derive a simpler closed-form $\bar{P}_{m d}^{\text {asy }}$ expression which does not contain infinite series sums and/or higher-order derivatives. Furthermore, the derived $\bar{P}_{m d}^{\text {asy }}$ unifies the analysis for fading channel models without diversity (Section V), with antenna diversity (Section VI), with cooperative diversity (Section VII), and in interference (Section VIII). Moreover, the excellent numerical match of our derived $\bar{P}_{m d}^{\text {asy }}$ with the exact results is reported in the corresponding sections. Next, we give one of our principal results in Proposition 3 below.

Proposition 3: Based on the proposed approximation $f^{a p p}(\beta)$, the average missed-detection probability $\bar{P}_{m d}^{\text {asy }}$ can be 
expressed as

$\bar{P}_{m d}^{\text {asy }}$
$= \begin{cases}1-a \Gamma(t+1) e^{-\lambda / 2} \sum_{i=1}^{2} \frac{1}{\left(\theta_{i}+\bar{\gamma}\right)^{t+1}}[\Psi(i)+\Phi(i)] & \text { for } u>t+1 \\ 1-a \Gamma(t+1) e^{-\lambda / 2} \sum_{i=1}^{2} \frac{1}{\left(\theta_{i}+\bar{\gamma}\right)^{t+1}} \Psi(i) & \text { for } u \leq t+1,\end{cases}$

with $\Psi(i)$ and $\Phi(i)$ given by

$$
\begin{aligned}
\Psi(i)=\frac{e^{\lambda \eta_{i} / 2}}{t !} \sum_{k=0}^{t}\left[\left(\begin{array}{l}
t \\
k
\end{array}\right) \frac{(-1)^{k} \prod_{j=1}^{k}(u-t+j-2)}{\eta_{i}^{u-t-1+k}}\right. & \\
& \left.\times \sum_{\nu=0}^{t-k} \frac{(\lambda / 2)^{t-k-v}}{\left(1-\eta_{i}\right)^{v+1}} \frac{(t-k) !}{(t-k-v) !}\right], \\
\Phi(i)=\frac{(-1)^{-(t+1)}}{(u-t-2) !} \sum_{k=0}^{u-t-2}\left[\left(\begin{array}{c}
u-t-2 \\
k
\end{array}\right) \frac{\prod_{j=1}^{k}(t+j)}{\eta_{i}^{t+k+1}}\right. & \left.\times \sum_{\nu=0}^{u-t-k-2} \frac{(\lambda / 2)^{u-t-k-2-v}(u-t-k-2) !}{(u-t-k-2-v) !}\right],
\end{aligned}
$$

with $\eta_{i}=\bar{\gamma} /\left(\theta_{i}+\bar{\gamma}\right), i=1,2$.

Proof: The proof is given in Appendix A.

At a sufficiently high $\operatorname{SNR}(\bar{\gamma} \gg 1), \bar{P}_{m d}^{\text {asy }}(16)$ reduces to the form $\bar{P}_{m d}^{\text {asy }} \approx 1-g(u, t, \lambda) \bar{\gamma}^{-(t+1)}$, where $g(u, t, \lambda)$ is a function independent of $\bar{\gamma}$. Clearly, the sensing gain, which is equal to the magnitude of the slope of the log-log plot of $\bar{P}_{m d}^{\text {asy }}$ vs. $\bar{\gamma}$ at high SNR, is given by magnitude of the exponent of $\bar{\gamma}$ occurring in $\bar{P}_{m d}^{\text {asy }}$, which is equal to $(t+1)$. This observation is consistent with the sensing gain given by [8]. Hence, our derived asymptotic explicitly reveals the sensing gain.

\section{A. Accuracy of (16)}

The accuracy of the derived $\bar{P}_{m d}^{\text {asy }}(16)$ and $\bar{P}_{m d}^{\text {wg }}$ (asymptotic $\bar{P}_{m d}$ of [8]) is depicted in terms of the log-log plots of the absolute error vs. $\bar{\gamma}$ in Fig. 2 for a 5-branch SC in i.i.d. Rayleigh fading channels. The exact PDF $f(\beta)(25)$ and the corresponding parameters of $f^{\text {app }}(\beta)$ are given in Section VI-B. The absolute errors for $\bar{P}_{m d}^{\text {asy }}$ and $\bar{P}_{m d}^{\mathrm{wg}}$ are defined as the difference $\mid \bar{P}_{m d}^{\text {asy }}-$ $\bar{P}_{m d}^{\text {exact }} \mid$ and $\left|\bar{P}_{m d}^{\mathrm{wg}}-\bar{P}_{m d}^{\text {exact }}\right|$, respectively, where $\bar{P}_{m d}^{\text {exact }}$ is computed from [2, eq. (30)]. At high SNR (say, $\bar{\gamma} \geq 14 \mathrm{~dB}$ ), the rate of decrease of the absolute error of $\bar{P}_{m d}^{\text {asy }}$ (slope $=-6$ ) is greater by an order of magnitude than that of $\bar{P}_{m d}^{\text {wg }}$ (slope $=-5$ ). As well, the absolute error of $\bar{P}_{m d}^{\text {asy }}$ is $10^{-5}$ or less for $\bar{\gamma} \geq 12.5 \mathrm{~dB}$ while $\bar{P}_{m d}^{\text {wg }}$ attains the same accuracy only for $\bar{\gamma} \geq 19 \mathrm{~dB}$. Therefore, the SNR gain of $\bar{P}_{m d}^{\text {asy }}$ in attaining an absolute error of $10^{-5}$ is at least $6.5 \mathrm{~dB}$ relative to $\bar{P}_{m d}^{\mathrm{wg}}$. Furthermore, for SNRs as low as $0 \mathrm{~dB},\left|\bar{P}_{m d}^{\text {asy }}-\bar{P}_{m d}^{\text {exact }}\right|$ is still very small $(\leq 0.1)$, while $\left|\bar{P}_{m d}^{\text {wg }}-\bar{P}_{m d}^{\text {exact }}\right|$ is far more than 1 (the breakdown mentioned in Section III-B). Thus, $\bar{P}_{m d}^{\text {asy }}$ is more accurate than $\bar{P}_{m d}^{\text {wg }}$ throughout the practical SNR range. This result can be attributed to our finding that $f^{\text {app }}(\beta)$ can indeed approximate the exact PDF more accurately for any $\beta>0$, unlike $f^{\mathrm{wg}}(\beta)$, which is accurate for mainly $\beta \rightarrow 0^{+}$(as discussed previously in the context of Fig. 1 in Section III-B).

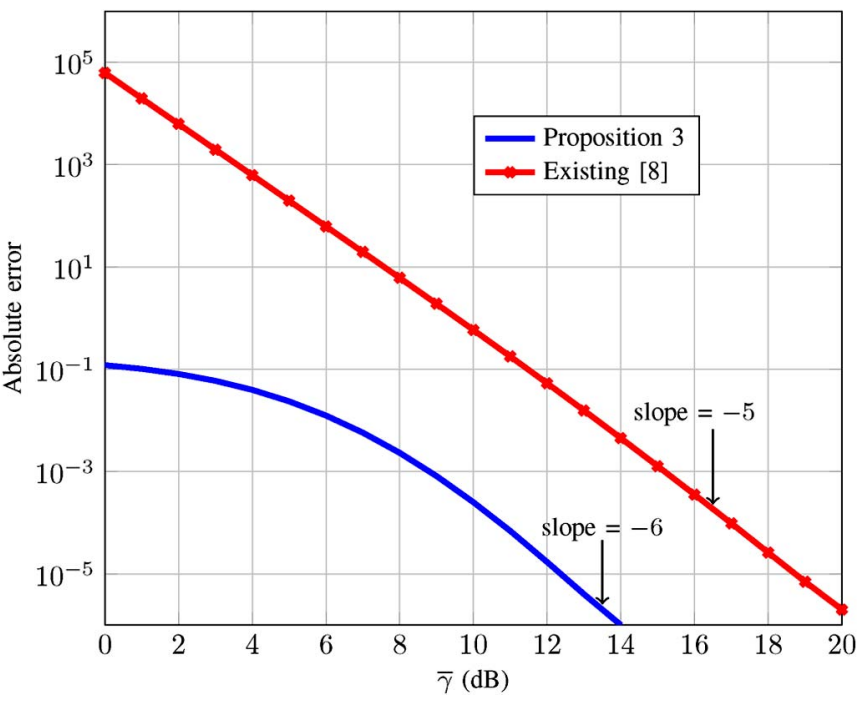

Fig. 2. Absolute error vs. SNR for a 5-branch SC in i.i.d. Rayleigh fading: Proposition 3 and [8].

Now that the accuracy of the derived $\bar{P}_{m d}^{\text {asy }}$ has been numerically verified, next we will assess the ED performance for various cases without and with antenna diversity reception, with cooperative detection, and in interference. We will first apply Proposition 1 or Proposition 2 (whichever most readily applies) to determine the parameters of $f^{\text {app }}(\beta)$ and then use Proposition 3 to compute $\bar{P}_{m d}^{\text {asy }}$. To evaluate the performance, we consider $\log$ - $\log$ plots of $\bar{P}_{m d}$ vs. $\bar{\gamma}$. The magnitude of the slopes of these plots at large SNR (say, $\bar{\gamma} \geq 20 \mathrm{~dB}$ ) yields the sensing gain. As well, since low false alarm probability is needed (for example, $P_{f} \leq 0.1$ for cognitive radio networks [16]), we fix $P_{f}=0.01$. The detection threshold $\lambda$ is then determined by solving (1) for the given $P_{f}$ and used for evaluating $\bar{P}_{m d}$.

\section{FADING Channels Without Diversity}

\section{A. Nakagami-m Fading}

One of the most versatile fading channel models, the Nakagami- $m$ distribution, fits empirical data and provides a better match than other models to some experimental data. For example, it accurately models land-mobile and indoor-mobile propagation environments [17]. For this model, the exact $f(\beta)$ is given by

$$
f(\beta)=\frac{m^{m}}{\Gamma(m)} \beta^{m-1} e^{-m \beta}, \beta>0
$$

where parameter $m \geq 1 / 2$ represents the fading severity index with the special case $m=1$ yielding the Rayleigh fading channel. By comparing (18) with (6), it is clear that $f^{\operatorname{app}}(\beta)$ exactly represents (18) when $a=m^{m} /[2 \Gamma(m)], t=m-1$ and $\theta_{1}=\theta_{2}=m$. Nevertheless, we will extract these parameters by using our Proposition 1 for verification.

Thus, performing the Maclaurin's series expansion of (18), we get

$$
f(\beta)=\frac{m^{m}}{\Gamma(m)} \beta^{m-1}-\frac{m^{m+1}}{\Gamma(m)} \beta^{m}+\frac{m^{m+2}}{2 \Gamma(m)} \beta^{m+1}+O\left(\beta^{m+2}\right) .
$$




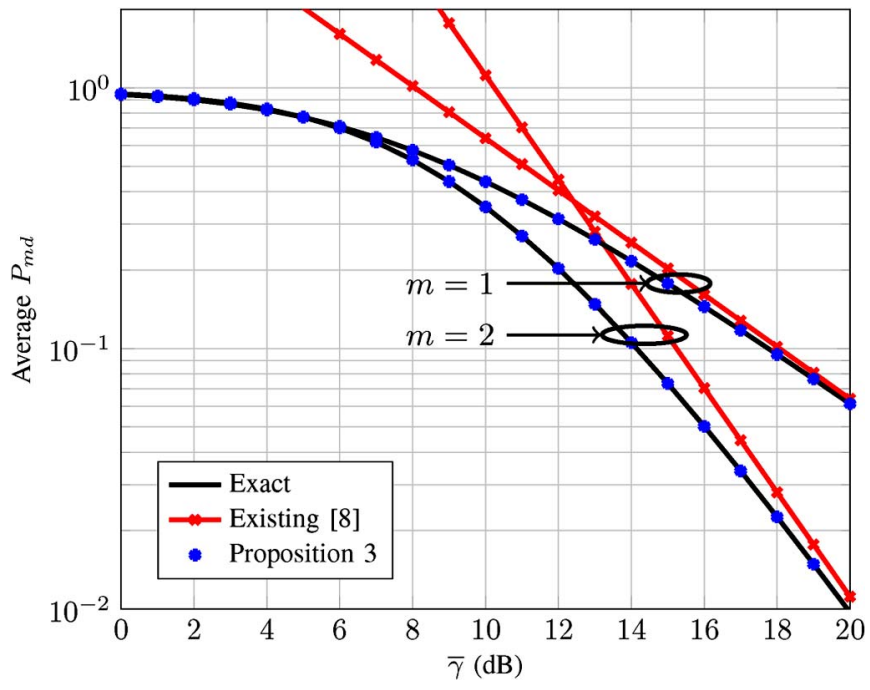

Fig. 3. $\bar{P}_{m d}$ vs. SNR in Nakagami- $m$ fading channel for $u=3$ : exact, existing [8] and Proposition 3.

Then, comparing (19) with (9) and using Proposition 1, we get $t=m-1, a=m^{m} /[2 \Gamma(m)], b_{1}=2 m$ and $b_{2}=2 m^{2}$. The substitution of $b_{1}$ and $b_{2}$ into (8) then gives $\theta_{1}=\theta_{2}=m$, as expected. This result validates our Proposition 1 .

Now that the parameters of $f^{\text {app }}(\beta)$ have been extracted, the use of Proposition 3 gives the desired expression for $\bar{P}_{m d}^{\text {asy }}$. In Fig. 3, our derived $\bar{P}_{m d}^{\text {asy }}(16)$ is compared against the exact $\bar{P}_{m d}$ computed from [5, eq. (5)] and against the asymptotic $\bar{P}_{m d}$ of [8]. As discussed in Section III-B, the asymptotic $\bar{P}_{m d}$ of [8] breaks down below a certain SNR. For instance, at $\bar{\gamma} \leq 8 \mathrm{~dB}$ for $m=1$, the asymptotic $\bar{P}_{m d}$ of [8] exceeds 1 . In contrast, our derived $\bar{P}_{m d}^{\text {asy }}$ does not suffer from such a drawback. In fact, $\bar{P}_{m d}^{\text {asy }}$ is virtually identical to the exact values computed from [5, eq. (5)] over the entire SNR range $(0 \leq \bar{\gamma} \mathrm{dB} \leq 20)$, whereas the asymptotic $\bar{P}_{m d}$ of [8] approaches the exact values only for $\bar{\gamma} \geq$ $19 \mathrm{~dB}$. Also, the exact $\bar{P}_{m d}$ computed from [5, eq. (5)] contains an infinite series, which requires series-truncation. Moreover, the number of terms in such series has to be experimentally determined for a given precision requirement for each set of parameters and must be updated whenever the parameters change. In contrast, our derived solution (16) does not require such computations. Finally, the magnitude of the slopes of the curves corresponding to $m=1$ and $m=2$ at high SNR (say, $\bar{\gamma} \geq 19 \mathrm{~dB}$ ) are observed to be 1 and 2 , respectively, thus verifying that the sensing gain is equal to $m(=t+1)$.

\section{B. Nakagami-q (Hoyt) Fading}

This fading is observed on satellite links suffering from strong ionospheric scintillation and spans from one-sided Gaussian $(q=0)$ to Rayleigh fading $(q=1)$ [17]. For the Nakagami- $q$ fading channel, the exact $f(\beta)$ is of the form

$$
f(\beta)=\frac{1+q^{2}}{2 q} e^{-\frac{(1+q)^{2}}{4 q^{2}} \beta} I_{0}\left(\frac{1-q^{4}}{4 q^{2}} \beta\right),
$$

where $I_{0}(\cdot)$ is the zero-th order modified Bessel function of the first kind. By Maclaurin's series expanding (20), grouping the

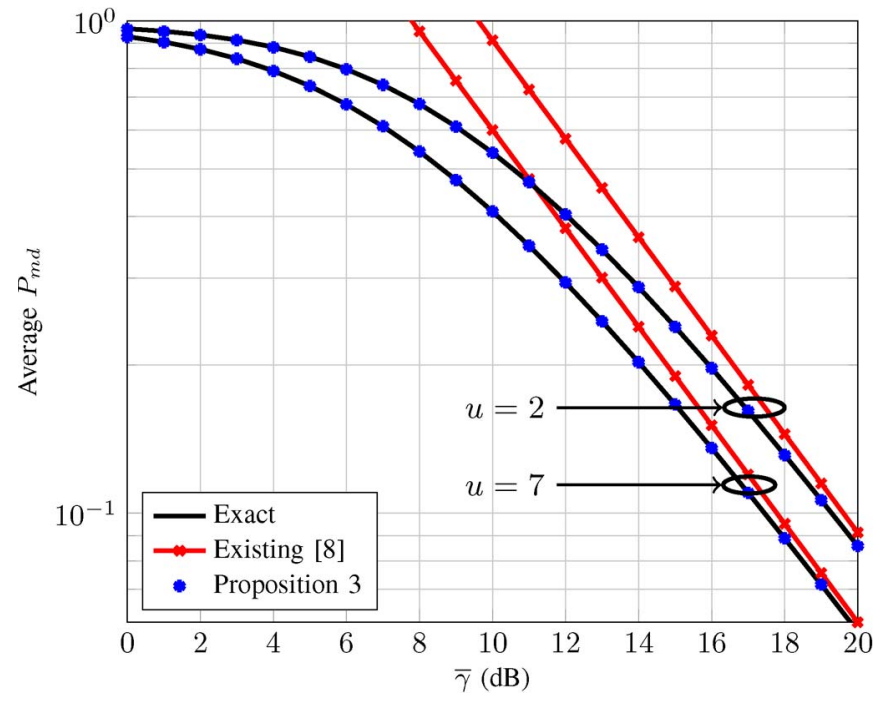

Fig. 4. $\bar{P}_{m d}$ vs. SNR in Nakagami- $q$ channel with $q=0.7$ for different $u$.

terms in ascending powers of $\beta$, and comparing the result with (4), we find that $t=0$ and

$$
\begin{aligned}
& a_{0}=\frac{1+q^{2}}{2 q}, a_{1}=-\frac{\left(1+q^{2}\right)}{2 q}\left(\frac{q^{4}+2 q^{2}+1}{4 q^{2}}\right), \\
& a_{2}=\frac{\left(1+q^{2}\right)}{2 q}\left(\frac{3 q^{8}+8 q^{6}+10 q^{4}+8 q^{2}+3}{64 q^{4}}\right) .
\end{aligned}
$$

Then, Proposition 1 readily yields $a, \theta_{1}$, and $\theta_{2}$, and Proposition 3 furnishes $\bar{P}_{m d}^{\text {asy }}$.

The comparative results for Nakagami- $q$ fading are depicted in Fig. 4. Clearly, our derived $\bar{P}_{m d}^{\text {asy }}$ is virtually exact (it has 7-digit precision) over the entire SNR range $(0 \leq \bar{\gamma} \mathrm{dB} \leq 20)$ while the asymptotic $\bar{P}_{m d}$ of [8] is accurate for $\bar{\gamma} \geq 19 \mathrm{~dB}$ only. Also, the magnitude of the slopes of the graphs at high SNR $(\bar{\gamma} \geq 19 \mathrm{~dB})$ are observed to be 1 (which equals the sensing gain $t+1)$.

\section{Fading Channels With Antenna Diversity}

Until now, only the single-antenna reception has been treated. Next, $L(\geq 1)$ antennas with mutually independent fading are considered. The parameters of $f^{\mathrm{app}}(\beta)$ for maximal ratio combining (MRC), equal gain combining (EGC), and SC are derived. The MRC and SC are coherent combining schemes which require channel-state information (CSI) at the ED. Although the CSI availability requirement contradicts the premise of ED (requiring no a priori information), these combining schemes are important for establishing the ED performance limits, a benchmark relative to which the performance of the alternative diversity combiners can be measured. Thus, the ED performance with antenna diversity combining assuming perfect CSI availability has been investigated extensively in the literature [2], [3], [5]-[7]. Moreover, as highlighted by [5] (and the references therein), for cognitive radio networks, the CSI may be available at the cognitive radios over control/broadcast channels (for example, by using low-rate pilot signal exchanges as shown in [18]). Nevertheless, since the purpose of this 
section is to demonstrate the applicability of our proposed analysis to antenna diversity combining, we proceed with the CSI availability notion for MRC and SC. On the other hand, EGC operates without any CSI.

\section{A. MRC With L Independent Antennas}

The optimal combining scheme in the absence of interference is the MRC which thus establishes an upper-bound performance for antenna diversity combining [17]. The MRC receiver combines all the diversity branches after weighting each branch with the complex conjugate of the corresponding fading channel coefficient to yield $y_{\mathrm{mrc}}(t)=\sum_{l=1}^{L} h_{l}^{*} y_{l}(t)$, where $y_{l}(t)$ is the received signal at the $l$-th branch, and $h_{l}^{*}$ is the complex conjugate of the $l$-th fading channel coefficient. The overall channel gain can then be expressed as $\beta=\sum_{l=1}^{L} \beta_{l}$, where $\beta_{1}, \beta_{2}, \ldots$, $\beta_{L}$ are statistically independent fading channel gains. Regardless of the fading channel model under consideration, if the MGF of each $\beta_{l}$ as $s \rightarrow \infty$ can be expressed in the form ${ }^{2}$

$$
\mathcal{M}_{\beta_{l}}(s)=\frac{c_{l}}{s^{\mu_{l}}}+\frac{d_{l}}{s^{\mu_{l}+1}}+\frac{e_{l}}{s^{\mu_{l}+2}}+O\left(\frac{1}{s^{\mu_{l}+3}}\right),
$$

then, the MGF of $\beta$ as $s \rightarrow \infty$ can be expressed as

$$
\mathcal{M}_{\beta}(s)=\prod_{l=1}^{L}\left[\frac{c_{l}}{s^{\mu_{l}}}+\frac{d_{l}}{s^{\mu_{l}+1}}+\frac{e_{l}}{s^{\mu_{l}+2}}+O\left(\frac{1}{s^{\mu_{l}+3}}\right)\right],
$$

which after some algebraic manipulations, can be expressed as (23), shown at the bottom of the page. Then, comparing (23) with (11) yields

$$
\begin{aligned}
\alpha & =\sum_{l=1}^{L} \mu_{l} ; X_{0}=\prod_{l=1}^{L} c_{l} ; X_{1}=\sum_{j=1}^{L} d_{j} \prod_{l=1, l \neq j}^{L} c_{l}, \\
X_{2} & =\sum_{j=1}^{L} e_{j} \prod_{l=1, l \neq j}^{L} c_{l}+\sum_{j=1}^{L-1} d_{j}\left(\sum_{k=j+1}^{L} d_{k} \prod_{l=1, l \neq k, l \neq j}^{L} c_{l}\right) .
\end{aligned}
$$

Thus, Proposition 2 readily gives the desired parameters $t, a$, $\theta_{1}$, and $\theta_{2}$, and Proposition 3 yields the corresponding $\bar{P}_{m d}^{\text {asy }}$.

To demonstrate the usefulness of (23), let us consider an example when the channel gains of the branches are independent and non-identically distributed (i.n.i.d.) Rayleigh random variables such that $f_{\beta_{l}}(x)=1 / l \cdot e^{-x / l}$. Then, the MGF for each $\beta_{l}$ is $\mathcal{M}_{\beta_{l}}(s)=1 /(1+l s)$. Performing the series expansion of $\mathcal{M}_{\beta_{l}}(s)$ as $s \rightarrow \infty$ and comparing the result with (22), we get $\mu_{l}=1, c_{l}=1 / l, d_{l}=1 / l^{2}$, and $e_{l}=1 / l^{3}$. Substituting these

${ }^{2}$ For the cases of practical interest (for example, multi-branch reception in Rayleigh fading, Nakagami- $m$ fading), the MGF of each branch can be written in the form (22). This is demonstrated with an example, shortly.

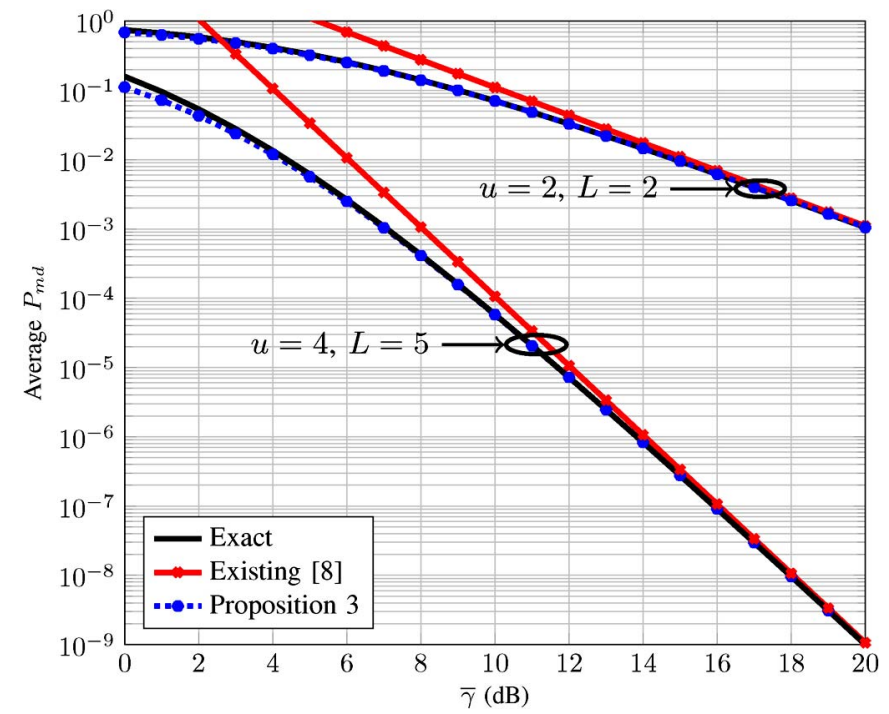

Fig. 5. $\bar{P}_{m d}$ vs. SNR for MRC in i.n.i.d. Rayleigh fading channels for different $\{u, L\}$.

coefficients in (24) immediately yields $\alpha, X_{0}, X_{1}$, and $X_{2}$. Then, the use of Proposition 2 followed by Proposition 3 gives $\bar{P}_{m d}^{\text {asy }}$.

The high accuracy of our derived $\bar{P}_{m d}^{\text {asy }}(16)$ over $0 \leq \bar{\gamma} \mathrm{dB} \leq 20$ is visible in Fig. 5 while the asymptotic $\bar{P}_{m d}$ of [8] is accurate only for say, $\bar{\gamma}>14 \mathrm{~dB}$. Also, the magnitude of the slopes of the graphs observed at $\bar{\gamma} \geq 14 \mathrm{~dB}$ are 2 and 5 , respectively, for $L=2$ and $L=5$. These results are equal to the corresponding sensing gains (thus verifying $t+1=L$ ).

\section{B. SC in Rayleigh Fading}

The SC is a reduced complexity scheme where only the branch with the largest SNR is selected and processed. For an $L$-branch SC in i.i.d. Rayleigh fading, the PDF of $\beta$ is given by

$$
f(\beta)=L e^{-\beta}\left(1-e^{-\beta}\right)^{L-1}, \beta \geq 0 .
$$

The Maclaurin's series expansion of (25) followed by some algebraic manipulations and comparison of the resulting expression with (4) yields $t=L-1, a_{i}=\mathcal{D}(L-1+i) /(L-1+i)$ ! for $i=0,1,2$, and $\mathcal{D}(\cdot)$ is defined as:

$$
\mathcal{D}(n)=L \sum_{k=0}^{L-1}\left(\begin{array}{c}
L-1 \\
k
\end{array}\right)(-1)^{k+n}(k+1)^{n} .
$$

Then, the use of Proposition 1 provides $a, \theta_{1}$, and $\theta_{2}$, and Proposition 3 subsequently yields $\bar{P}_{m d}^{\text {asy }}$. The graphs in Fig. 6 yet again depict the remarkable accuracy of our $\bar{P}_{m d}^{\text {asy }}(16)$ compared against the asymptotic $\bar{P}_{m d}$ of [8] and the exact $\bar{P}_{m d}$ obtained from $[2$, eq. (30)]. The observed sensing gain from all the

$$
\mathcal{M}_{\beta}(s)=\frac{\prod_{l=1}^{L} c_{l}}{s^{\sum_{l} \mu_{l}}}+\frac{\sum_{j=1}^{L} d_{j} \prod_{l=1, l \neq j}^{L} c_{l}}{s^{\sum_{l=1}^{L} \mu_{l}+1}}+\frac{\sum_{j=1}^{L} e_{j} \prod_{l=1, l \neq j}^{L} c_{l}+\sum_{j=1}^{L-1} d_{j}\left(\sum_{k=j+1}^{L} d_{k} \prod_{l=1, l \neq k, l \neq j}^{L} c_{l}\right)}{s^{\sum_{l=1}^{L} \mu_{l}+2}}+O\left(\frac{1}{s_{l=1}^{L} \mu_{l}+3}\right)
$$




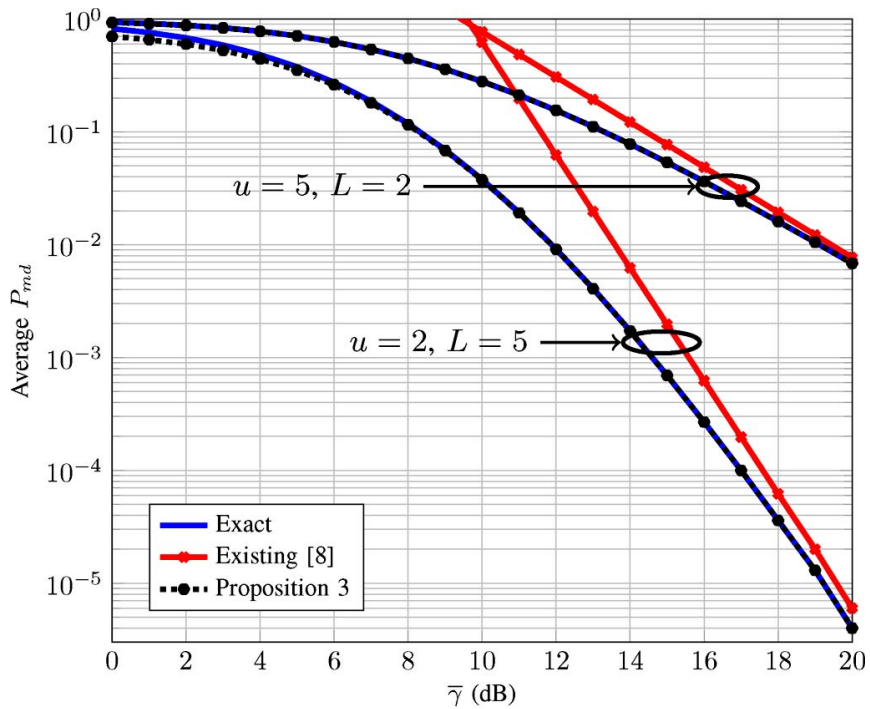

Fig. 6. $\bar{P}_{m d}$ vs. SNR for SC in i.i.d. Rayleigh fading.

graphs at high SNR $(\bar{\gamma} \geq 15 \mathrm{~dB})$ is clearly equal to $L$ (consistent with $t+1=L$ ).

\section{EGC and SC in Nakagami-m Fading}

EGC, unlike MRC, does not require estimation of the fading amplitudes and hence has a lower complexity than MRC [19]. For a 2-branch EGC in Nakagami- $m$ fading, the exact $f(\beta)$ can be obtained from [5, eq. (32)] to be

$$
\begin{aligned}
f(\beta)=\frac{2^{2-2 m} \sqrt{\pi} \Gamma(2 m) m^{2 m}}{\Gamma^{2}(m) \Gamma\left(2 m+\frac{1}{2}\right)} & \beta^{2 m-1} e^{-2 m \beta} \\
& \times{ }_{1} F_{1}\left(2 m ; 2 m+\frac{1}{2} ; m \beta\right),
\end{aligned}
$$

where ${ }_{1} F_{1}(\cdot ; \cdot ; \cdot)$ is the confluent hypergeometric function [20]. Maclaurin's series expansion of (27) followed by comparison with (4) gives $t=2 m-1, \quad a_{0}=\left[2^{2-2 m} \sqrt{\pi} \Gamma(2 m) m^{2 m}\right] /$ $\left[\Gamma^{2}(m) \Gamma(2 m+1 / 2)\right], a_{1}=-\left[2 m(2 m+1) a_{0}\right] /[4 m+1], a_{2}=$ $\left[2 m^{2}\left(4 m^{2}+6 m+3\right) a_{0}\right] /[(4 m+1)(4 m+3)]$. Then, use of Proposition 1 and Proposition 3 subsequently yields the desired $\bar{P}_{m d}^{\text {asy }}$. Note that we consider the 2-branch EGC case here because the PDFs of SNR for the $L(>2)$-branch EGC in Nakagami- $m$ fading do not have a closed-form and rather contain one or more infinite series (see [5, eq. (40)], [5, eq. (45)]) for which the number of terms needed for obtaining a desired precision (accuracy) is unknown. Thus, the parameters of $f^{\text {app }}(\beta)$ for such cases will be in terms of infinite series, and the number of terms needed for precise truncation of the infinite series is unknown.

Next, we consider 3-branch SC in Nakagami- $m$ fading. Using [5, eq. (50)] with $L=3$, we can write the exact PDF of $\beta$ to be

$$
f(\beta)=\frac{3 m^{m}}{\Gamma^{3}(m)} \beta^{m-1} e^{-m \beta}[\mathcal{G}(m, m \beta)]^{2},
$$

where $\mathcal{G}(a, z)=\int_{0}^{z} x^{a-1} e^{-x} d x$ is the lower incomplete Gamma function [13]. Maclaurin's series expansion of $f(\beta)$ gives $t=$ $3 m-1, a_{0}=\left[3 m^{3 m-2}\right] /\left[\Gamma^{3}(m)\right], a_{1}=-\left[3 m^{3 m-1}(2 m+1)\right] /$ $\left[(m+1) \Gamma^{3}(m)\right]$ and $a_{2}=\left[3 m^{3 m}\left(9 m^{3}+24 m^{2}+15 m+2\right)\right] /$

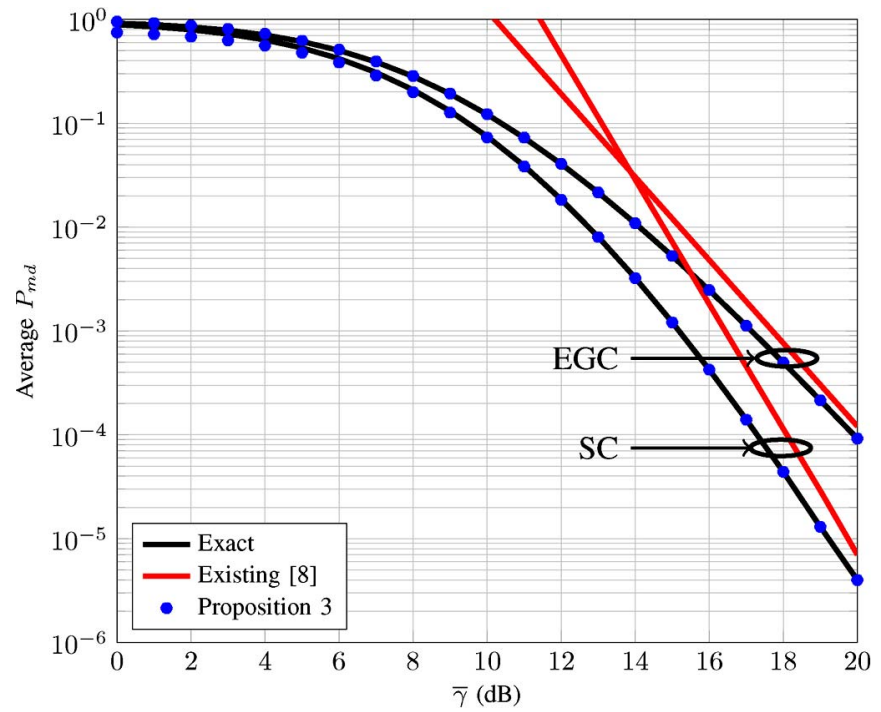

Fig. 7. $\bar{P}_{m d}$ vs. SNR for 2-branch EGC $(u=5)$ and 3-branch SC $(u=2)$ in Nakagami-2 fading.

$\left[2 \Gamma^{3}(m)(m+1)^{2}(m+2)\right]$. Then, the use of Proposition 1 followed by Proposition 3 subsequently gives $\bar{P}_{m d}^{\text {asy }}$. It is worth noting that $\mathrm{SC}$ cases with $L \geq 4$ can be treated similarly because the exact $f(\beta)$ (obtained using [5, eq. (50)]) can still be Maclaurin's series expanded in the form (9), however, at the expense of additional algebraic manipulations. Nevertheless, those cases are not treated any further for brevity.

The comparative results for the 2-branch EGC and 3-branch $\mathrm{SC}$ in Nakagami-2 fading are depicted in Fig. 7 where the benefit of our $\bar{P}_{m d}^{\text {asy }}$ (16) over the asymptotic $\bar{P}_{m d}$ of [8] is clear. The exact $\bar{P}_{m d}$ for EGC and SC are obtained by using [5, eq. (38)] and [5, eq. (59)], respectively, which are infinite series expressions involving the hypergeometric functions and thus the sensing gains from such expressions are not explicit. Unlike the use of these equations, application of Proposition 1 clearly suggests a sensing gain of $2 m(=4)$ for EGC and $3 m(=6)$ for SC, respectively (given by slopes of the graphs at, say, $\bar{\gamma} \geq 19 \mathrm{~dB}$ ).

\section{Cooperative Detection With Multiple Relays}

In the previous section, the performance of an ED equipped with co-located multiple antennas has been characterized for several antenna diversity combining schemes. Although the benefits of co-located multiple antennas are widely accepted, deploying them may be impractical in situations where size, cost, or hardware pose limitations. Examples include handsets (where size is a limitation) and low-power, small-size nodes in a wireless sensor network [21]. In these scenarios, a different form of spatial diversity can be more practical. In this form, a number of wireless nodes at different locations independently relay their received signal (which is the desired signal at the destination receiver) to a central (destination) receiver. Moreover, in the context of signal detection, such cooperation among multiple relays and the central receiver gives rise to a cooperative detection network where the central receiver, called the fusion center (FC), combines the signals forwarded by the relays and makes a final decision on the presence/absence of the SUT. Such a set-up yields a significant gain in detection performance 
compared to that of the system with a single detector [22]. We thus consider a cooperative detection network where a number $(n \geq 1)$ of relays amplify-and-forward their received signals (which are multiple copies of the transmit SUT) to the FC, which then makes the final decision on the presence/absence of the SUT based on these $n$ observations. This scenario fits, for example, in the context of cooperative spectrum sensing with cognitive relay networks. Furthermore, the FC may have access to CSI of the channels from the primary user (PU) to the relays and from relays to the FC. This may be possible via channel estimation at the relays through the use of low-rate pilot signals [18] and control channel signalling [23].

The total instantaneous SNR $\gamma$ of such a network can be upper-bounded by $\gamma_{\text {up }}$ as $\gamma \leq \gamma_{\text {up }}=\sum_{i=1}^{n} \gamma_{i}^{\text {min }}$, where $\gamma_{i}^{\text {min }}=$ $\min \left\{\gamma_{p r_{i}}, \gamma_{r_{i}}\right\} ; \gamma_{p r_{i}}$ and $\gamma_{r_{i} d}$ are the instantaneous SNRs of the links from the transmitter to the $i$-th relay $r_{i}$ and from $r_{i}$ to the FC, respectively [24]. ${ }^{3}$ Assume the channels from each relay to the FC are statistically independent Rayleigh fading. The MGF of $\gamma_{\text {up }}, \mathcal{M}_{\gamma_{\text {up }}}(s)$, is given by [24, eq. (10)]

$$
\mathcal{M}_{\gamma_{\text {up }}}(s)=\prod_{i=1}^{n} \frac{\bar{\gamma}_{p r_{i}}+\bar{\gamma}_{r_{i} d}}{\bar{\gamma}_{p r_{i}} \bar{\gamma}_{r_{i} d}} \cdot \frac{1}{\left(s+\frac{\bar{\gamma}_{p r_{i}+\bar{\gamma}_{r_{i} d}}}{\bar{\gamma}_{p r_{i}} \bar{\gamma}_{r_{i} d}}\right)},
$$

where $\bar{\gamma}_{p r_{i}}$ and $\bar{\gamma}_{r_{i} d}$ are the average SNRs of the respective links. In general, the series expansion of the MGF (29) at $s \rightarrow \infty$ would result in MGF of the form (23), which was derived for the MRC with $L$ independent antennas. Without loss of generality and for the sake of brevity, we omit similar details, and, instead, provide an example where the link SNRs are identical, i.e., $\bar{\gamma}_{p r_{i}}=\bar{\gamma}_{r_{i} d}=\bar{\gamma}$. This set-up allows (29) to be expressed in the form

$$
\mathcal{M}_{\gamma_{\text {up }}}(s)=\frac{(2 / \bar{\gamma})^{n}}{s^{n}[1+2 /(\bar{\gamma} s)]^{n}} .
$$

We define $\gamma_{\text {up }}=\bar{\gamma} \beta_{\text {up }}$. Then, by using the transformation of the random variables, the MGF of $\beta_{\text {up }}$ can be obtained from that of $\gamma_{\text {up }}$ as $\mathcal{M}_{\beta_{\text {up }}}(s)=\mathcal{M}_{\gamma_{\text {up }}}(s / \bar{\gamma})$. Performing a series expansion of the resulting expression for $\mathcal{M}_{\beta_{\text {up }}}(s)$ at $s \rightarrow \infty$ gives

$$
\mathcal{M}_{\beta_{\text {up }}}(s)=\frac{2^{n}}{s^{n}}-\frac{n 2^{n+1}}{s^{n+1}}+\frac{n(n+1) 2^{n+1}}{s^{n+2}}+O\left(\frac{1}{s^{n+3}}\right) .
$$

Then, applying Proposition 2, we get $\alpha=n, X_{0}=2^{n}, X_{1}=$ $-n 2^{n+1}$ and $X_{2}=n(n+1) 2^{n+1}$. These findings readily yield the parameters $t, a, \theta_{1}$ and $\theta_{2}$. Thus, Proposition 3 can be applied to obtain $\bar{P}_{m d}^{\text {asy }}$. The results comparing the exact $\bar{P}_{m d}$ (based on $\gamma_{\text {up }}$ ) obtained from [24, eq. (11), the asymptotic $\bar{P}_{m d}$ of [8], and our derived $\bar{P}_{m d}^{\text {asy }}(16)$ are depicted in Fig. 8. Clearly, these results indicate the benefit of our derived $\bar{P}_{m d}^{\text {asy }}$ over the asymptotic $\bar{P}_{m d}$ of [8]. Also, the magnitude of the slopes of the graphs at high SNR $(\geq 19 \mathrm{~dB})$ are found to be same as the number of cooperating relays $n$, which is equal to the sensing gain $(t+1=n)$.

\footnotetext{
${ }^{3}$ Since use of the exact MGF of $\gamma$ to obtain a closed-form $\bar{P}_{m d}$ is analytically intractable [24], we use $\gamma_{\text {up }}$ instead, which can lead to a closed-form upper bound for $\bar{P}_{m d}$.
}

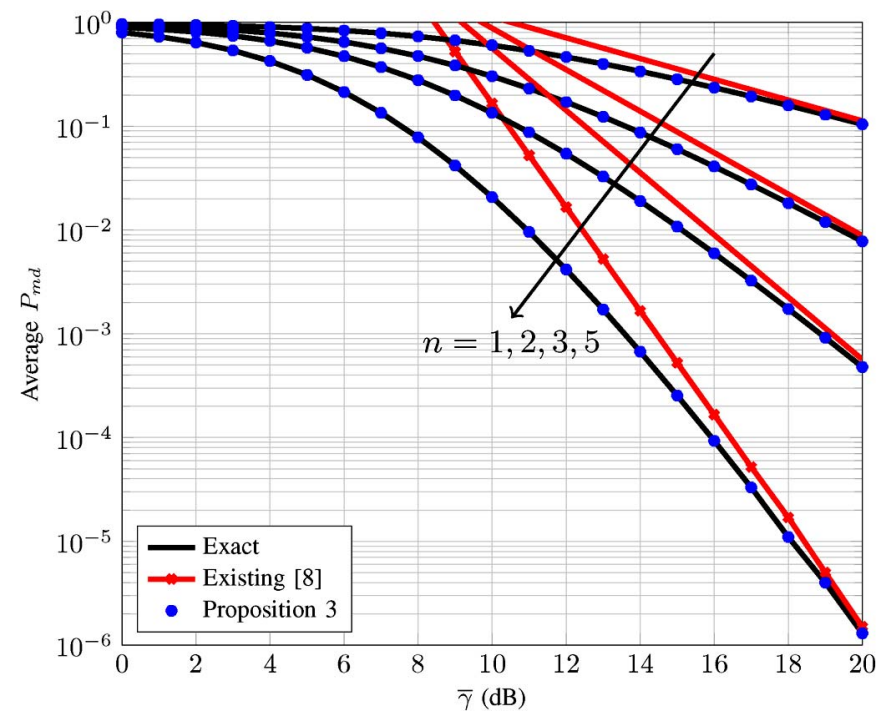

Fig. 8. $\bar{P}_{m d}$ vs. SNR for multiple relays based detection $(u=2)$.

\section{DeteCtion In InTERFERENCE}

In the previous sections, as in existing works on ED performance analysis [2]-[8], [24], the presence of interfering transmissions has been disregarded. In emerging broadband technologies (such as LTE-Advanced), small cells (pico- and femto-cells) are commonly deployed by overlaying them over the traditional macrocells of cellular networks, thus extending the wireless coverage and capacity [25]. This set-up results in a heterogeneous network consisting of a large number of wireless nodes such that the reception (at the destination) of undesired transmissions (that have leaked in space over relatively large distances) occurs inevitably [26]. Thus, all the heterogeneous nodes sharing the same spectrum suffer from interference. In such situations, an ED equipped node will receive interfering transmissions, which will deteriorate its detection performance [27].

Thus, in the presence of interference, the received signal (at the ED) is composed of the faded version of the SUT, numerous interfering signals from other users, and additive noise (which is generally assumed to be white Gaussian). In this situation, rather than SNR, the signal-to-interference-plusnoise ratio (SINR) has to be taken into account, which is defined (with abuse of the notation $\gamma$ ) as

$$
\gamma=\frac{h_{0} P_{0}}{\sum_{i=1}^{N_{I}} h_{i} P_{i}+N_{0}},
$$

where $N_{0}$ is noise variance, $h_{0}$ and $P_{0}$ are the desired transmitter-to-receiver channel gain and the average power of the desired signal, respectively, while $h_{i}$ and $P_{i}, \forall i \in\{1,2, \ldots$, $\left.N_{I}\right\}$, are the channel gain and average power of the $i$-th interfering link, respectively, with $N_{I}$ being the total number of interferers. We assume all the channels undergo Rayleigh fading. Furthermore, the detector is equipped with $L \geq 1$ antennas, whose signals are combined by using MRC. Hence, SINR can still be expressed as $\gamma=\bar{\gamma} \beta$, where $\bar{\gamma}=P_{0} /\left(I_{\text {total }}+N_{0}\right)$ is the average SINR with $I_{\text {total }}=\sum_{i=1}^{N_{I}} P_{i}$ and $\beta=h_{0}\left(I_{\text {total }}+N_{0}\right) /$ 


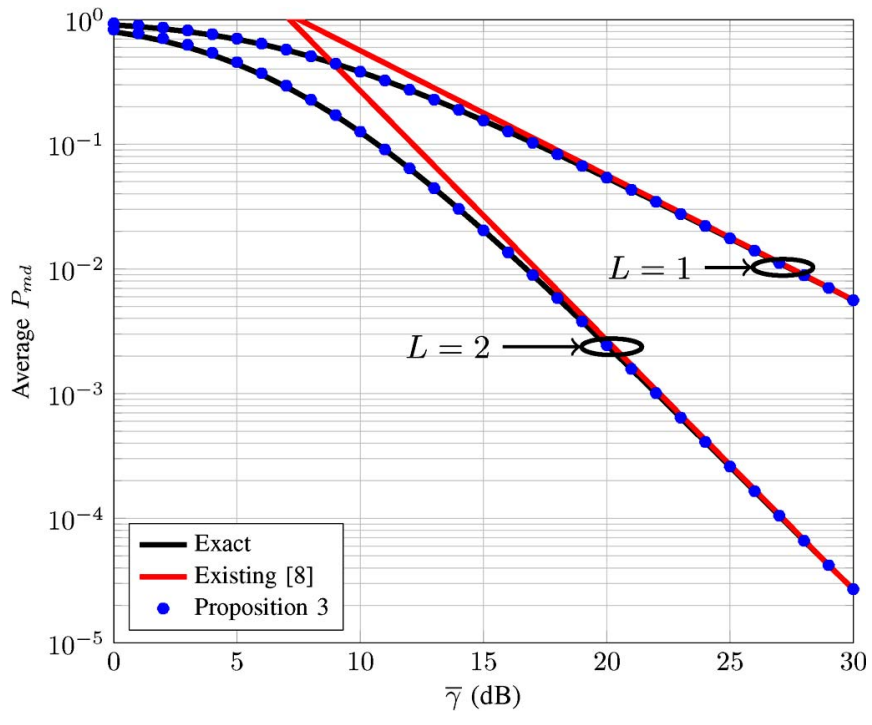

Fig. 9. $\bar{P}_{m d}$ vs. $\bar{\gamma}$ for $u=2$ in presence of $N_{I}=8$ interferers with powers $\left\{P_{1}, P_{2}, P_{3}, P_{4}, P_{5}, P_{6}, P_{7}, P_{8}\right\}=\{-5,-3,-1,1,3,5,7,9\} \mathrm{dB}, N_{0}=0 \mathrm{~dB}$.

$\left[\sum_{i=1}^{N_{I}} P_{i} h_{i}+N_{0}\right]$. This set-up leads to the following PDF of $\beta[28]$ :

$$
f(\beta)=\frac{\beta^{L-1}}{(L-1) !} \sum_{i=1}^{N_{I}} \frac{\mathcal{A}_{i} e^{\mathcal{C}_{i}} \mathcal{B}_{i}^{L}}{\left(1+\mathcal{B}_{i} \beta\right)^{L+1}} \Gamma\left(L+1, \mathcal{C}_{i}\left(1+\mathcal{B}_{i} \beta\right)\right),
$$

where $\mathcal{A}_{i}=\prod_{k=1, k \neq i}^{N_{I}} P_{i} /\left(P_{i}-P_{k}\right), \mathcal{B}_{i}=P_{i} /\left[I_{\text {total }}+N_{0}\right]$, and $\mathcal{C}_{i}=$ $N_{0} / P_{i}$. With the abuse of notations, we use $\beta$ and $\bar{\gamma}$ here for presentation simplicity and for notational consistency. Then, the Maclaurin's series expansion of (31) yields $t=L-1, a_{0}=$ $\sum_{i=1}^{N_{I}} \mathcal{A}_{i} \mathcal{B}_{i}^{L} e^{\mathcal{C}_{i}} \Gamma\left(L+1, \mathcal{C}_{i}\right), \quad a_{1}=-\sum_{i=1}^{N_{I}} \mathcal{A}_{i} \mathcal{B}_{i}^{L+1}\left[\mathcal{C}_{i}^{L+1}+e^{\mathcal{C}_{i}} \times\right.$ $\left.(L+1) \Gamma\left(L+1, \mathcal{C}_{i}\right)\right]$, and $a_{2}=\sum_{i=1}^{N_{I}} \frac{1}{2} \mathcal{A}_{i} \mathcal{B}_{i}^{L+2}\left[e^{\mathcal{C}_{i}}\left(L^{2}+3 L+\right.\right.$ 2) $\left.\Gamma\left(L+1, \mathcal{C}_{i}\right)+\mathcal{C}_{i}^{L+1}\left\{2+2 L-e^{\mathcal{C}_{i}} \Gamma(L+1){ }_{1} \tilde{F}_{1}\left(L+1 ; L ;-\mathcal{C}_{i}\right)\right\}\right]$, where ${ }_{1} \tilde{F}_{1}(\cdot ; \cdot ; \cdot)$ is the regularized confluent hypergeometric function of the confluent hypergeometric function ${ }_{1} F_{1}(\cdot ; \cdot ; \cdot)$ [29]. Thus, Proposition 1, and subsequently, Proposition 3 can be used to obtain the desired parameters and $\bar{P}_{m d}^{\text {asy }}$, respectively.

A numerical example for this case is depicted in Fig. 9, which indicates significantly better accuracy of our derived $\bar{P}_{m d}^{\text {asy }}$ over $0 \leq \bar{\gamma} \mathrm{dB} \leq 30$, in contrast to the asymptotic $\bar{P}_{m d}$ of [8], which is accurate only for say, $\bar{\gamma} \geq 18 \mathrm{~dB}$. Furthermore, the sensing gains are equal to the number of antennas $L$ (the magnitude of the slopes of the graphs at $\bar{\gamma} \geq 20 \mathrm{~dB}$ are equal to 1 and 2 for $L=1$ and $L=2$, respectively).

\section{Average CAUC}

Thus far, we have considered the problem of averaging the Marcum- $Q$ function over $f^{\mathrm{app}}(\beta)$ (Proposition 3). However, we emphasize that the application of $f^{\text {app }}(\beta)$ goes beyond Proposition 3. Its wider applications could include analyses of the outage probability, bit error rates, average capacity, and symbol error rates, which are critical performance metrics of practical digital wireless systems. However, due to the page limitation, we omit these analyses. Nevertheless, we will consider one more application, the analysis of CAUC for ED.

Although the well-known ROC curves (the plots of $P_{d}$ vs. $P_{f}$ ) graphically represent the ED performance, a single figure of merit for concisely representing the ED's overall detection capability is desirable. For this purpose, the area under the ROC curve (AUC), which varies between $1 / 2$ and 1 , was recently proposed [11]. If the AUC is $1 / 2$, then the decision is as reliable as that of a coin toss, meaning $P_{m d}=0.5$. Thus, a larger AUC implies a better detector. However, neither the ROC curves nor the AUC graphs explicitly reveal the order of improvement in detection performance with increasing SNR. This shortcoming led to the introduction of yet another single figure of merit, CAUC, which reveals the order of improvement in detection performance with increasing SNR, explicitly. The instantaneous CAUC for ED is given by [30]

$$
\begin{aligned}
A^{\prime}(\gamma)=\sum_{k=0}^{u-1} \frac{1}{2^{k} k !} \gamma^{k} e^{-\frac{\gamma}{2}} & -\sum_{k=1-u}^{u-1} \frac{\Gamma(u+k)}{2^{u+k} \Gamma(u)} \\
& \times e^{-\gamma} \tilde{F}_{1}\left(u+k ; k+1 ; \frac{\gamma}{2}\right) .
\end{aligned}
$$

Intuitively, a lower CAUC implies a better detection capability.

We are interested in evaluating the average asymptotic CAUC, denoted by $\overline{A^{\prime}}$ asy, which can be obtained by integrating $\left.A^{\prime}(\gamma)\right|_{\gamma=\bar{\gamma} \beta}$ over $f^{\text {app }}(\beta)$ as

$$
{\overline{A^{\prime}}}_{\text {asy }}=\int_{0}^{\infty} A^{\prime}(\bar{\gamma} \beta) f^{\text {app }}(\beta) d \beta,
$$

which can be derived to be (34) (see Appendix C for derivation details),

$$
\begin{aligned}
{\overline{A^{\prime}}}_{\text {asy }}= & a \sum_{k=0}^{u-1} \frac{\bar{\gamma}^{k}}{2^{k} k !} \Gamma(t+k+1) \sum_{i=1}^{2} \frac{1}{\left(\theta_{i}+\bar{\gamma} / 2\right)^{t+k+1}} \\
& -a \sum_{k=1-u}^{u-1} \frac{\Gamma(u+k) \Gamma(t+1)}{2^{u+k} \Gamma(u)} \sum_{i=1}^{2} \frac{1}{\left(\theta_{i}+\bar{\gamma}\right)^{t+1}} \\
& \times{ }_{2} \tilde{F}_{1}\left(t+1, u+k ; k+1 ; \frac{\bar{\gamma}}{2\left(\theta_{i}+\bar{\gamma}\right)}\right)
\end{aligned}
$$

where ${ }_{2} \tilde{F}_{1}(\cdot, \cdot ; \cdot ; \cdot)$ is the regularized confluent hypergeometric function of the confluent hypergeometric function ${ }_{2} F_{1}(\cdot, \cdot ; \cdot ; \cdot)$ [31]. At large SNRs $(\bar{\gamma} \gg 1),(34)$ reduces to the form $\bar{A}_{\text {asy }} \approx$ $g_{\text {auc }}(u, t) \bar{\gamma}^{-(t+1)}$, where $g_{\text {auc }}(u, t)$ is a term independent of $\bar{\gamma}$. Thus, magnitude of the exponent of $\bar{\gamma}$ occurring in $\overline{A^{\prime}}$ asy at large SNRs is equal to $(t+1)$, which in fact is the sensing gain obtained from our derived asymptotic missed-detection probability $\bar{P}_{m d}^{\text {asy }}(16)$ at high SNR. The same exponent is equivalently defined as the "detection diversity gain order" in [30]. Hence, the sensing gain is also given by the magnitude of the slope of the log-log plot of $\overline{A^{\prime}}$ asy vs. $\bar{\gamma}$ at high SNR.

A numerical example for the average CAUC (denoted by $\overline{A^{\prime}}$ ) for SC in i.i.d. Rayleigh fading (for which the parameters of $f^{\text {app }}(\beta)$ were derived in Section VI-B) is shown in Fig. 10 where our derived asymptotic CAUC $\overline{A^{\prime}}$ asy $(34)$ is compared against the approximate and exact CAUCs computed by using the approximation $f^{\mathrm{wg}}(\beta)$ (5) and the exact $f(\beta)(25)$, respectively. Interestingly, our $\overline{A^{\prime}}$ asy is virtually identical to the exact values over the entire SNR range $(0 \leq \bar{\gamma} \mathrm{dB} \leq 20)$, while $\overline{A^{\prime}}$ computed by using $f^{\mathrm{wg}}(\beta)$ is accurate for only $\bar{\gamma} \geq 19 \mathrm{~dB}$. Furthermore, the sensing gains obtained from the graphs at high $\operatorname{SNR}(\bar{\gamma} \geq$ $19 \mathrm{~dB}$ ) are 2 and 4 for $L=2$ and $L=4$, respectively (thus verifying $t+1=L)$. Thus, the proposed $f^{\text {app }}(\beta)$ serves as a 


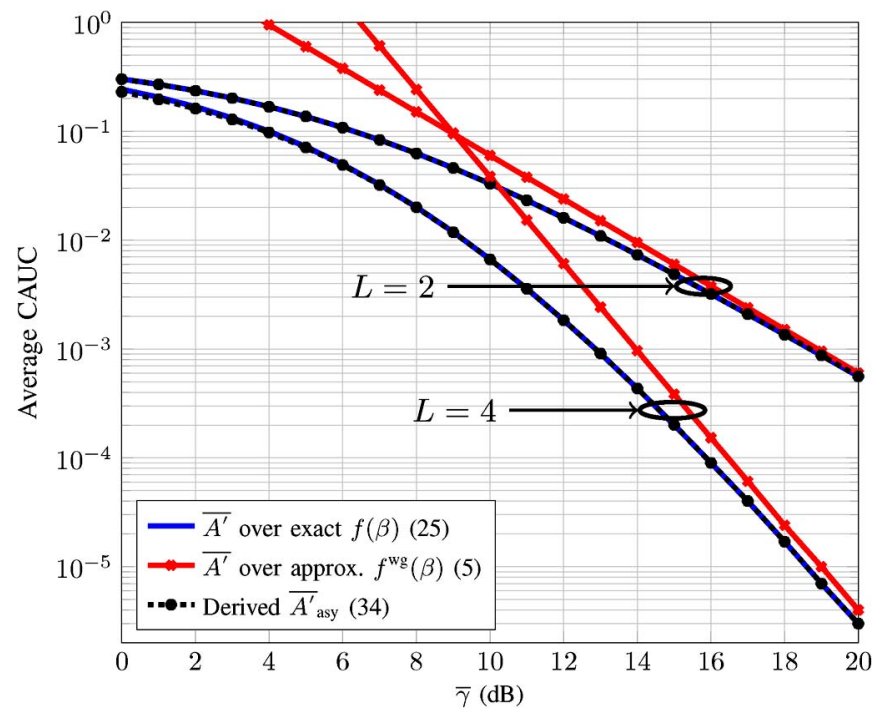

Fig. 10. $\overline{A^{\prime}}$ vs. SNR for SC in i.i.d. Rayleigh fading with $u=2$.

unified approximation for the exact PDF of $\beta$, which can also be applied for deriving the average performance metrics from the instantaneous ones involving functions other than the Marcum$Q$ function.

\section{Conclusion}

In this paper, a new approximation $f^{\text {app }}(\beta)$ with parameters matching the operating conditions (the fading channel, antenna diversity, cooperative diversity, among others) is proposed. These parameters are obtained using the exact PDF or MGF of $\beta$. By utilizing the MGF corresponding to $f^{\operatorname{app}(\beta)}$ along with the contour integral representation of the Marcum- $Q$ function, the unified asymptotic missed-detection probability $\bar{P}_{m d}^{\text {asy }}$ of the ED is derived in closed-form. The derived $\bar{P}_{m d}^{\text {asy }}$ is then specialized to single-antenna systems, antenna diversity, multiple-relay-based cooperative detection, and to interference environments. Our $\bar{P}_{m d}^{\text {asy }}$ is found to be highly accurate (achieving up to 7-digit precision for some cases), and valid over a wider SNR range than that derived by using the approximation in [8]. Thus, compared with [8], although our approximation requires only a simple additional step for extracting more information, this step improves both the accuracy and valid SNR range of the asymptotic analysis. Moreover, the derived $\bar{P}_{m d}^{\text {asy }}$ also explicitly reveals the sensing gain. To show another application of our proposed $f^{\text {app }}(\beta)$, we also derive the asymptotic CAUC, the result being highly accurate, and additionally revealing the sensing gain. Thus, we have developed a unified comprehensive analytical framework to characterize the ED performance in different practical communication scenarios. This framework may subsequently help in designing robust detectors in state-of-the-art and future wireless communication networks.

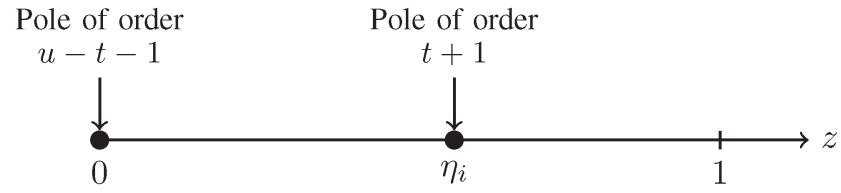

Fig. 11. Possible poles of $g_{i}(z), i=1,2$, in the $z$-plane.

In this work, we consider $f^{\text {app }}(\beta)$ for analyzing ED performance only. However, the application of our proposed $f^{\text {app }}(\beta)$ to analyze other crucial metrics such as the symbol error rate, outage probability, or channel capacity (which are useful for characterizing wireless systems deploying space-time coding and modulations, multiple-input and multiple-output technology, antenna/relay selection schemes, and numerous other techniques [32]-[36]) may be of interest to wireless communication researchers and engineers. Thus, this paper opens up interesting and diverse future research opportunities.

\section{APPENDIX}

\section{A. Derivation of $\bar{P}_{m d}^{a s y}(16)$}

Substituting the contour integral representation for the Marcum- $Q$ function given by [15, eq. (1)] into (3), we get

$$
\bar{P}_{m d}=1-\frac{e^{-\frac{\lambda}{2}}}{2 \pi J} \oint_{\Delta} \mathcal{M}_{\gamma}\left(1-\frac{1}{z}\right) \frac{e^{\frac{\lambda}{2} z}}{z^{u}(1-z)} d z,
$$

where $\Delta$ is a circular contour of radius $r$ such that $0<r<1$, and $J$ denotes the imaginary unit. The MGF of $\gamma, \mathcal{M}_{\gamma}(s)$, in (35) can be expressed in terms of the MGF of $\beta$ as $\mathcal{M}_{\gamma}(s)=$ $\mathcal{M}_{\beta}(s \bar{\gamma})$. Then, substituting the MGF $\mathcal{M}_{\beta}^{\mathrm{app}}(s)$ from (14) into the resulting expression, and following some algebraic manipulations, we get

$$
\bar{P}_{m d}^{\text {asy }}=1-\frac{a \Gamma(t+1) e^{-\frac{\lambda}{2}}}{2 \pi J} \oint_{\Delta}\left[g_{1}(z)+g_{2}(z)\right] d z,
$$

where $g_{i}(z)=\frac{1}{\left(\theta_{i}+\bar{\gamma}\right)^{t+1}} \frac{e^{\lambda z / 2}}{\left(z-\eta_{i}\right)^{t+1} z^{u-t-1}(1-z)}$, for $i=1$, 2, with $\eta_{i} \triangleq \bar{\gamma} /\left(\theta_{i}+\bar{\gamma}\right)$. As $g_{i}(z)$, for $i=1,2$, may contain a pole of order $(u-t-1)$ at $z=0$ (for $u>t+1)$ and a pole of order $(t+1)$ at $z=\eta_{i}$ (see Fig. 11), in order to evaluate the contour integral in (36), we need the residues of $g_{i}(z)$ at these poles. Thus, two cases arise as follows.

Case I: $u>t+1:$ In this case, the function $g_{i}(z), i=1,2$, contains a $(t+1)$-th ordered pole at $z=\eta_{i}$ and a $(u-t-1)$-th ordered pole at $z=0$.

Case II: $u \leq t+1$ : For this case, $g_{i}(z), i=1,2$, does not have any pole at $z=0$, and only the pole at $z=\eta_{i}$ contributes to the contour integral.

The two cases thus lead to $\bar{P}_{m d}^{\text {asy }}$ of the form (37), shown at the bottom of the page, where the notation $\operatorname{Res}\left[g(z) ; z_{0}, p\right]$ denotes

$$
\bar{P}_{m d}^{\text {asy }}= \begin{cases}1-a \Gamma(t+1) e^{-\frac{\lambda}{2}} \sum_{i=1}^{2} \operatorname{Res}\left[g_{i}(z) ; \eta_{i}, t+1\right]+\operatorname{Res}\left[g_{i}(z) ; 0, u-t-1\right] & \text { for } u>t+1 \\ 1-a \Gamma(t+1) e^{-\frac{\lambda}{2}} \sum_{i=1}^{2} \operatorname{Res}\left[g_{i}(z) ; \eta_{i}, t+1\right] & \text { for } u \leq t+1\end{cases}
$$


the residue of the function $g(z)$ at pole $z=z_{0}$ of order $p$. This residue is defined as [37]

$$
\left.\operatorname{Res}\left[g(z) ; z_{0}, p\right] \triangleq \frac{1}{(p-1) !} \frac{d^{p-1}}{d z^{p-1}}\left[g(z)\left(z-z_{0}\right)^{p}\right]\right|_{z=z_{0}} .
$$

The residue $\operatorname{Res}\left(g_{i} ; \eta_{i}, t+1\right), i=1,2$, can thus be expressed in closed-form as (see Appendix B)

$\operatorname{Res}\left[g_{i}(z) ; \eta_{i}, t+1\right]=\frac{1}{\left(\theta_{i}+\bar{\gamma}\right)^{t+1} t !} \sum_{k=0}^{t}\left(\begin{array}{l}t \\ k\end{array}\right) \psi_{\eta_{i}}^{(k)} \phi_{\eta_{i}}^{(t-k)}$,

where $\psi_{\eta_{i}}^{(k)}$ is the $k$-th order derivative of $\psi(z) \triangleq 1 /\left(z^{u-t-1}\right)$ with respect to $z$, and $\phi_{\eta_{i}}^{(t-k)}$ is the $(t-k)$-th order derivative of $\phi(z) \triangleq e^{\lambda z / 2} /(1-z)$ with respect to $z$, both evaluated at $z=\eta_{i}$, which are given by

$$
\begin{aligned}
\psi_{\eta_{i}}^{(k)} & =\frac{(-1)^{k}}{\eta_{i}^{u-t-1+k}} \prod_{j=1}^{k}(u-t+j-2), \\
\phi_{\eta_{i}}^{(t-k)} & =e^{\frac{\lambda}{2} \eta_{i}} \sum_{\nu=0}^{n} \frac{(\lambda / 2)^{n-v}}{\left(1-\eta_{i}\right)^{\nu+1}} \frac{n !}{(n-v) !} .
\end{aligned}
$$

By following the steps similar to those used in deriving $\operatorname{Res}\left[g_{i}(z) ; \eta_{i}, t+1\right], \operatorname{Res}\left[g_{i}(z) ; 0, u-t-1\right], i=1,2$, can be obtained to be (details omitted for the sake of brevity)

$$
\begin{aligned}
& \operatorname{Res}\left(g_{i}(z) ; 0, u-t\right.-1)=\frac{1}{\left(\theta_{i}+\bar{\gamma}\right)^{t+1}(u-t-2) !} \\
& \times \sum_{k=0}^{u-t-2}\left(\begin{array}{c}
u-t-2 \\
k
\end{array}\right) \chi_{0}^{(k)} \phi_{0}^{(u-t-k-2)},
\end{aligned}
$$

where $\chi_{0}^{(k)}$ is the $k$-th order derivative of $\chi(z) \triangleq 1 /\left(z-\eta_{i}\right)^{t+1}$ with respect to $z$, evaluated at $z=0$ and can be derived to be

$$
\chi_{0}^{(k)}=\frac{(-1)^{-(t+1)}}{\eta_{i}^{t+k+1}} \prod_{j=1}^{k}(t+j)
$$

and $\phi_{0}^{(u-t-k-2)}$ is the same as $\phi_{\eta_{i}}^{(t-k)}$ with $(t-k)$ and $\eta_{i}$ replaced by $(u-t-k-2)$ and 0 , respectively. Then, using (39)-(43) in (37) results in (16).

\section{B. Derivation of (39)}

By applying the definition of the residue given in (38), $\operatorname{Res}\left[g_{i}(z) ; \eta_{i}, t+1\right], i=1,2$, can be expressed as

$$
\operatorname{Res}\left[g_{i}(z) ; \eta_{i}, t+1\right]=\left.\frac{1}{\left(\theta_{i}+\bar{\gamma}\right)^{t+1} t !} \frac{d^{t}}{d z^{t}}[\psi(z) \phi(z)]\right|_{z=\eta_{i}} .
$$

Then, utilizing the general Leibniz rule for finding the $t$-th order derivative of a product [13, eq. (3.3.8)] on the right-hand side of (44), followed by evaluating the resulting expression at $z=\eta_{i}$ gives:

$$
\left.\frac{d^{t}}{d z^{t}}[\psi(z) \phi(z)]\right|_{z=\eta_{i}}=\sum_{k=0}^{t}\left(\begin{array}{l}
t \\
k
\end{array}\right) \psi_{\eta_{i}}^{(k)} \phi_{\eta_{i}}^{(t-k)} .
$$

Thus, substituting (45) into (44) yields (39). The coefficients $\psi_{\eta_{i}}^{(k)}$ of (45) can be derived by mathematical induction to be as in (40). For deriving $\phi_{\eta_{i}}^{(t-k)}$, the Leibniz rule can be re-applied after expressing $\phi(z)$ as a product of the terms $e^{\lambda z / 2}$ and $1 /(1-z)$ and then, following some algebraic manipulations, to yield (41).

\section{Derivation of ${\overline{A^{\prime}}}_{\text {asy }}$ (34)}

By substituting (6) into (33), the average CAUC over $f^{\text {app }}(\beta)$ can be expressed as

$$
{\overline{A^{\prime}}}_{\text {asy }}=1-a \sum_{k=0}^{u-1} \frac{\bar{\gamma}^{k}}{2^{k} k !} \mathcal{I}_{1}+a \sum_{k=1-u}^{u-1} \frac{\Gamma(u+k)}{2^{u+k} \Gamma(u)} \mathcal{I}_{2},
$$

where $\mathcal{I}_{1}$ is defined as

$$
\mathcal{I}_{1} \triangleq \sum_{i=1}^{2} \int_{0}^{\infty} \beta^{t+k} e^{-\left(\theta_{i}+\frac{\bar{\gamma}}{2}\right) \beta} d \beta=\sum_{i=1}^{2} \frac{\Gamma(t+k+1)}{\left(\theta_{i}+\bar{\gamma} / 2\right)^{t+k+1}},
$$

where the definition of Gamma function $\Gamma(\cdot)$ is used to solve the integral, and $\mathcal{I}_{2}$ is defined as

$\mathcal{I}_{2} \triangleq \sum_{i=1}^{2} \int_{0}^{\infty} \beta^{t} \tilde{F}_{1}\left(u+k ; k+1 ; \frac{\bar{\gamma}}{2} \beta\right) e^{-\left(\theta_{i}+\bar{\gamma}\right) \beta} d \beta=\sum_{i=1}^{2} \mathcal{I}_{2, i}$

To solve the integral $\mathcal{I}_{2, i}, i=1,2$, in (48), we proceed as follows. Given the generalized hypergeometric function ${ }_{p} F_{q}\left(a_{1}\right.$, $\left.\ldots, a_{p} ; b_{1}, \ldots, b_{q} ; z\right)$, the corresponding regularized hypergeometric function is given by [38] (with the abuse of notation) as

$$
\begin{aligned}
{ }_{p} \tilde{F}_{q}\left(a_{1}, \ldots, a_{p} ; b_{1}, \ldots, b_{q} ; z\right) & =\frac{{ } F_{q}\left(a_{1}, \ldots, a_{p} ; b_{1}, \ldots, b_{q} ; z\right)}{\Gamma\left(b_{1}\right) \ldots \Gamma\left(b_{q}\right)} \\
& =\sum_{v=0}^{\infty} \frac{\prod_{j=1}^{p}\left(a_{j}\right)_{v} z^{v}}{v ! \prod_{j=1}^{q} \Gamma\left(v+b_{j}\right)},
\end{aligned}
$$

where $\left(a_{j}\right)_{v}=\Gamma\left(a_{j}+v\right) / \Gamma\left(a_{j}\right)$ is the Pochammer's symbol. Then, using (49) with $p=q=1$ and substituting the resulting series expression into $\mathcal{I}_{2, i}$, we get $\mathcal{I}_{2, i}={ }_{2} \tilde{F}_{1}(t+1, k+u ; k+1$; $\left.\frac{\bar{\gamma}}{2\left(\theta_{i}+\bar{\gamma}\right)}\right) /\left(\bar{\gamma}+\theta_{i}\right)^{t+1}$, where the definition of Gamma function $\Gamma(\cdot)$ is applied to solve the resulting integral followed by the use of (49) for $p=2, q=1$. Thus, substituting $\mathcal{I}_{2, i}$ into (48) gives

$$
\mathcal{I}_{2}=\sum_{i=1}^{2} \frac{1}{\left(\bar{\gamma}+\theta_{i}\right)^{t+1}}{ }_{2} \tilde{F}_{1}\left(t+1, k+u ; k+1 ; \frac{\bar{\gamma}}{2\left(\theta_{i}+\bar{\gamma}\right)}\right) \text {. }
$$

Then, substituting (47) and (50) into (46) yields $\overline{A^{\prime}}$ asy (34).

\section{REFERENCES}

[1] S. Atapattu, C. Tellambura, and H. Jiang, Energy Detection for Spectrum Sensing in Cognitive Radio. New York, NY, USA: Springer-Verlag, 2014.

[2] F. F. Digham, M.-S. Alouini, and M. K. Simon, "On the energy detection of unknown signals over fading channels," in Proc. IEEE ICC, May 2003, pp. 3575-3579

[3] F. F. Digham, M.-S. Alouini, and M. K. Simon, "On the energy detection of unknown signals over fading channels," IEEE Trans. Commun., vol. 55, no. 1, pp. 21-24, Jan. 2007. 
[4] V. Kostylev, "Energy detection of a signal with random amplitude," in Proc. IEEE ICC, 2002, pp. 1606-1610.

[5] S. P. Herath, N. Rajatheva, and C. Tellambura, "Energy detection of unknown signals in fading and diversity reception," IEEE Trans. Commun., vol. 59, no. 9, pp. 2443-2453, Sep. 2011.

[6] A. Annamalai, O. Olabiyi, S. Alam, O. Odejide, and D. Vaman, "Unified analysis of energy detection of unknown signals over generalized fading channels," in Proc. IWCMC, Jul. 2011, pp. 636-641.

[7] O. Olabiyi and A. Annamalai, "Further results on the performance of energy detector over generalized fading channels," in Proc. IEEE Int. Symp. PIMRC, Sep. 2011, pp. 604-608.

[8] Q. Wang and D.-W. Yue, "A general parameterization quantifying performance in energy detection," IEEE Signal Process. Lett., vol. 16, no. 8, pp. 699-702, Aug. 2009.

[9] Z. Wang and G. Giannakis, "A simple and general parameterization quantifying performance in fading channels," IEEE Trans. Commun., vol. 51, no. 8, pp. 1389-1398, Aug. 2003

[10] IEEE Standard for Local and Metropolitan Area Networks Part 16: Air Interface for Broadband Wireless Access Systems, IEEE Std. 802.16-2009, 2009.

[11] S. Atapattu, C. Tellambura, and H. Jiang, "Analysis of area under the ROC curve of energy detection," IEEE Trans. Wireless Commun., vol. 9, no. 3, pp. 1216-1225, Mar. 2010.

[12] A. H. Nuttall, "Some integrals involving the $Q$ function," Naval Underwater Syst. Center (NUSC), Newport, RI, USA, Tech. Rep., Apr. 1972.

[13] M. Abramowitz and I. A. Stegun, Handbook of Mathematical Functions With Formulas, Graphs and Mathematical Tables. Gaithersburg, MD, USA: Nat. Bureau Standards, Nov. 1970.

[14] Y. Dhungana and C. Tellambura, "New simple approximations for error probability and outage in fading," IEEE Commun. Lett., vol. 16, no. 11, pp. 1760-1763, Nov. 2012.

[15] C. Tellambura, A. Annamalai, and V. Bhargava, "Closed form and infinite series solutions for the MGF of a dual-diversity selection combiner output in bivariate Nakagami fading," IEEE Trans. Commun., vol. 51, no. 4, pp. 539-542, Apr. 2003.

[16] C. Stevenson et al., "IEEE 802.22: The first cognitive radio wireless regional area network standard," IEEE Commun. Mag., vol. 47, no. 1, pp. 130-138, Jan. 2009.

[17] M. K. Simon and M.-S. Alouini, Digital Communication Over Fading Channels, 2nd ed. New York, NY, USA: Wiley, 2005.

[18] Y. Lu and A. Duel-Hallen, "Channel-adaptive sensing strategy for cognitive radio ad hoc networks," in Proc. IEEE Consum. Commun. Netw. Conf., Jan 2013, pp. 466-471.

[19] A. Annamalai, C. Tellambura, and V. Bhargava, "Equal-gain diversity receiver performance in wireless channels," IEEE Trans. Commun., vol. 48, no. 10 , pp. 1732-1745, Oct. 2000.

[20] Wolfram. [Online]. Available: http://functions.wolfram.com/ HypergeometricFunctions/Hypergeometric1F1/

[21] A. Nosratinia, T. Hunter, and A. Hedayat, "Cooperative communication in wireless networks," IEEE Commun. Mag., vol. 42, no. 10, pp. 74-80, Oct. 2004

[22] K. Letaief and W. Zhang, "Cooperative communications for cognitive radio networks," in Proc. IEEE, vol. 97, no. 5, pp. 878-893, May 2009.

[23] A. Bletsas, A. Lippnian, and D. Reed, "A simple distributed method for relay selection in cooperative diversity wireless networks, based on reciprocity and channel measurements," in Proc. IEEE VTC-Spring, May/Jun. 2005, pp. 1484-1488.

[24] S. Atapattu, C. Tellambura, and H. Jiang, "Energy detection based cooperative spectrum sensing in cognitive radio networks," IEEE Trans. Wireless Commun., vol. 10, no. 4, pp. 1232-1241, Apr. 2011.

[25] A. Ghosh, R. Ratasuk, B. Mondal, N. Mangalvedhe, and T. Thomas, "LTE-advanced: Next-generation wireless broadband technology," IEEE Wireless Commun., vol. 17, no. 3, pp. 10-22, Jun. 2010.

[26] J. Andrews, R. Ganti, M. Haenggi, N. Jindal, and S. Weber, "A primer on spatial modeling and analysis in wireless networks," IEEE Commun. Mag., vol. 48, no. 11, pp. 156-163, Nov. 2010.

[27] A. Makarfi and K. Hamdi, "Interference analysis of energy detection for spectrum sensing," IEEE Trans. Veh. Technol., vol. 62, no. 6, pp. 25702578, Jul. 2013.

[28] Y. Tokgoz and B. Rao, "The effect of imperfect channel estimation on the performance of maximum ratio combining in the presence of cochannel interference," IEEE Trans. Veh. Technol., vol. 55, no. 5, pp. 1527-1534, Sep. 2006.

[29] Wolfram. [Online]. Available: http://functions.wolfram.com/ HypergeometricFunctions/Hypergeometric1F1Regularized/02/
[30] S. Atapattu, C. Tellambura, and H. Jiang, "Performance of energy detection: A complementary AUC approach," in Proc. IEEE GLOBECOM, Dec. 2010, pp. 1-5.

[31] Wolfram. [Online]. Available: http://functions.wolfram.com/ HypergeometricFunctions/Hypergeometric2F1Regularized/09/

[32] M. Ramezani, M. Hajiaghayi, C. Tellambura, and M. Ardakani, "Receive antenna selection for unitary space-time modulation over semi-correlated Ricean channels," IEEE Trans. Commun., vol. 58, no. 2, pp. 521-530, Feb. 2010.

[33] G. Amarasuriya, C. Tellambura, and M. Ardakani, "Performance analysis framework for transmit antenna selection strategies of cooperative MIMO AF relay networks," IEEE Trans. Veh. Technol., vol. 60, no. 7, pp. 3030-3044, Sep. 2011.

[34] G. Amarasuriya, M. Ardakani, and C. Tellambura, "Output-threshold multiple-relay-selection scheme for cooperative wireless networks," IEEE Trans. Veh. Technol., vol. 59, no. 6, pp. 3091-3097, Jul. 2010.

[35] O. Amin, S. Ikki, and M. Uysal, "On the performance analysis of multirelay cooperative diversity systems with channel estimation errors," IEEE Trans. Veh. Technol., vol. 60, no. 5, pp. 2050-2059, Jun. 2011.

[36] A. Nezampour and R. Schober, "Asymptotic analysis of space-time codes in generalized fading channels," IEEE Commun. Lett., vol. 13, no. 8, pp. 561-563, Aug. 2009.

[37] R. P. Agarwal, K. Perera, and S. Pinelas, An Introduction to Complex Analysis. New York, NY, USA: Springer-Verlag, 2011.

[38] Wolfram. [Online]. Available: http://functions.wolfram.com/ HypergeometricFunctions/HypergeometricPFQRegularized/

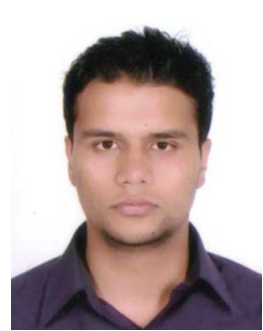

Vesh Raj Sharma Banjade (S'14) received the B.Eng. degree in electronics and communications engineering from the Institute of Engineering, Pulchowk Campus, Nepal, in 2009, and the M.Eng. degree in telecommunications engineering from the Asian Institute of Technology (AIT), Pathumthani, Thailand in 2011. He is now pursuing the Ph.D. degree at the Department of Electrical and Computer Engineering, University of Alberta, Edmonton, AB, Canada. His research interests include physical layer aspects of broadband wireless communication systems, spatial diversity techniques, and cognitive radio communications. He is currently an Intern at the Intel Labs.

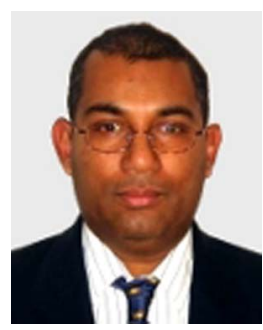

Chintha Tellambura (F'11) received the B.Sc. degree (with first-class honor) from the University of Moratuwa, Moratuwa, Sri Lanka, in 1986, the M.Sc. degree in electronics from the University of London, London, U.K., in 1988, and the Ph.D. degree in electrical engineering from the University of Victoria, Victoria, BC, Canada, in 1993.

He was a Postdoctoral Research Fellow with the University of Victoria, Victoria, BC, (1993-1994) and the University of Bradford, Bradford, West Yorkshire, U.K., (1995-1996). He was with Monash University, Clayton, Vic., Australia, from 1997 to 2002. Presently, he is a Professor with the Department of Electrical and Computer Engineering, University of Alberta. His research interests focus on communication theory dealing with the wireless physical layer.

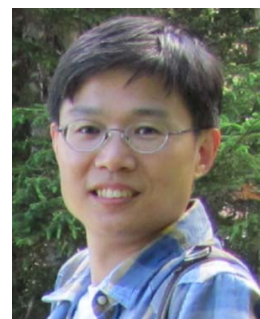

Hai Jiang (SM'15) received the B.Sc. and M.Sc. degrees in electronics engineering from Peking University, Beijing, China, in 1995 and 1998, respectively, and the Ph.D. degree in electrical engineering from the University of Waterloo, Waterloo, ON, Canada, in 2006. He is currently an Associate Professor at the Department of Electrical \& Computer Engineering, University of Alberta, Edmonton, AB, Canada. His research interests include radio resource management, cognitive radio networking, and cooperative communications. 